\title{
Decitabine alters the expression of Mecp2 isoforms via dynamic DNA methylation at the Mecp2 regulatory elements in neural stem cells
}

Vichithra R B Liyanage, Robby M Zachariah and Mojgan Rastegar*

\begin{abstract}
Background: Aberrant MeCP2 expression in brain is associated with neurodevelopmental disorders including autism. In the brain of stressed mouse and autistic human patients, reduced MeCP2 expression is correlated with Mecp2/MECP2 promoter hypermethylation. Altered expression of MeCP2 isoforms (MeCP2E1 and MeCP2E2) is associated with neurological disorders, highlighting the importance of proper regulation of both isoforms. While known regulatory elements (REs) within the MECP2/Mecp2 promoter and intron 1 are involved in MECP2/Mecp2 regulation, Mecp2 isoform-specific regulatory mechanisms are unknown. We hypothesized that DNA methylation at these REs may impact the expression of Mecp2 isoforms.

Methods: We used a previously characterized in vitro differentiating neural stem cell (NSC) system to investigate the interplay between Mecp2 isoform-specific expression and DNA methylation at the Mecp2 REs. We studied altered expression of Mecp2 isoforms, affected by global DNA demethylation and remethylation, induced by exposure and withdrawal of decitabine (5-Aza-2'-deoxycytidine). Further, we performed correlation analysis between DNA methylation at the Mecp2 REs and the expression of Mecp2 isoforms after decitabine exposure and withdrawal.
\end{abstract}

Results: At different stages of NSC differentiation, Mecp2 isoforms showed reciprocal expression patterns associated with minor, but significant changes in DNA methylation at the Mecp2 REs. Decitabine treatment induced Mecp2e1/ MeCP2E1 (but not Mecp2e2) expression at day (D) 2, associated with DNA demethylation at the Mecp2 REs. In contrast, decitabine withdrawal downregulated both Mecp2 isoforms to different extents at D8, without affecting DNA methylation at the Mecp2 REs. NSC cell fate commitment was minimally affected by decitabine under tested conditions. Expression of both isoforms negatively correlated with methylation at specific regions of the Mecp2 promoter, both at D2 and D8. The correlation between intron 1 methylation and Mecp2e1 (but not Mecp2e2) varied depending on the stage of NSC differentiation (D2: negative; D8: positive).

Conclusions: Our results show the correlation between the expression of Mecp2 isoforms and DNA methylation in differentiating NSC, providing insights on the potential role of DNA methylation at the Mecp2 REs in Mecp2 isoform-specific expression. The ability of decitabine to induce Mecp2e1/MeCP2E1, but not Mecp2e2 suggests differential sensitivity of Mecp2 isoforms to decitabine and is important for future drug therapies for autism.

Keywords: Epigenetics, Mecp2e1, Mecp2e2, Decitabine/5-aza-2'-deoxycytidine, DNA methylation, Autism

\footnotetext{
* Correspondence: rastegar@cc.umanitoba.ca

Regenerative Medicine Program, Department of Biochemistry and Medical

Genetics, Faculty of Medicine, University of Manitoba, Rm. 627, Basic Medical

Sciences Bldg., 745 Bannatyne Avenue, Winnipeg, Manitoba R3E 0J9, Canada
} 


\section{Background}

Methyl CpG Binding Protein 2 (MeCP2) is a key transcriptional regulator in the brain [1]. MECP2 mutations and expression deficits result in a broad range of neurodevelopmental disorders, including Rett syndrome (RTT) and autism spectrum disorders [2,3]. In mice (Mecp2) and humans (MECP2), alternative splicing of a single gene leads to the generation of two protein isoforms MeCP2E1 and MeCP2E2 (mature transcripts for Mecp2e1 and Mecp $2 e 2$ are shown in Figure 1A) [4,5]. We and others have shown differential expression of the two Mecp2/ $\mathrm{MeCP} 2$ isoforms in mouse brain [5-7]. Recent studies suggest that MeCP2E1 is the most relevant isoform for RTT pathology [8,9]. Moreover, overexpression of Mecp2e2, but not Mecp2e1, promotes neuronal cell death [10], implicating the importance of proper regulation of both Mecp2 isoforms in the brain.

In RTT mouse models, transgenic expression of either Mecp 2 isoform can rescue RTT phenotypes to different extents [14,15]. However, gene therapy delivery of $M E C P 2$ into the affected cells or drug therapies to induce $M E C P 2$ expression has to be carried out with caution, as even mild overexpression of MeCP2 can lead to progressive neurological disorders [16,17]. Currently, limited knowledge exists on $M E C P 2 / M e c p 2$ regulation, with no specific knowledge on possible differential $M E C P 2 / M e c p 2$ isoform-specific regulatory mechanisms.

$M E C P 2 / M e c p 2$ gene expression is known to be regulated by regulatory elements (REs) within the promoter and a silencer element within the Mecp2 intron 1 $[12,13,18]$ (Figure 1B). Implying the role of DNA methylation in $M E C P 2$ regulation, reduced $M E C P 2$ expression in the brains of male autistic patients correlates with human $M E C P 2$ promoter hypermethylation [2,19]. Moreover, reduced $M e c p 2$ expression in the postnatal murine brain in response to early maternal separation and stress is associated with hypermethylation of the mouse Mecp2 promoter [20]. However, possible differential impact of DNA methylation on $M E C P 2 / M e c p 2$ isoforms is currently unknown. DNA methylation is a major epigenetic modification that controls gene expression without affecting the underlying DNA sequences (reviewed in $[21,22])$. DNA methylation at the cytosine residues (5methylcytosine $(5 \mathrm{mC})$ ) of the $\mathrm{CpG}$ dinucleotides is carried out by DNA methyltransferases (DNMT) and is generally considered to be a repressive epigenetic modification [1,23]. Conversely, 5-hydroxymethylcytosine (5hmC), which is generated by oxidation of $5 \mathrm{mC}$ by TET proteins

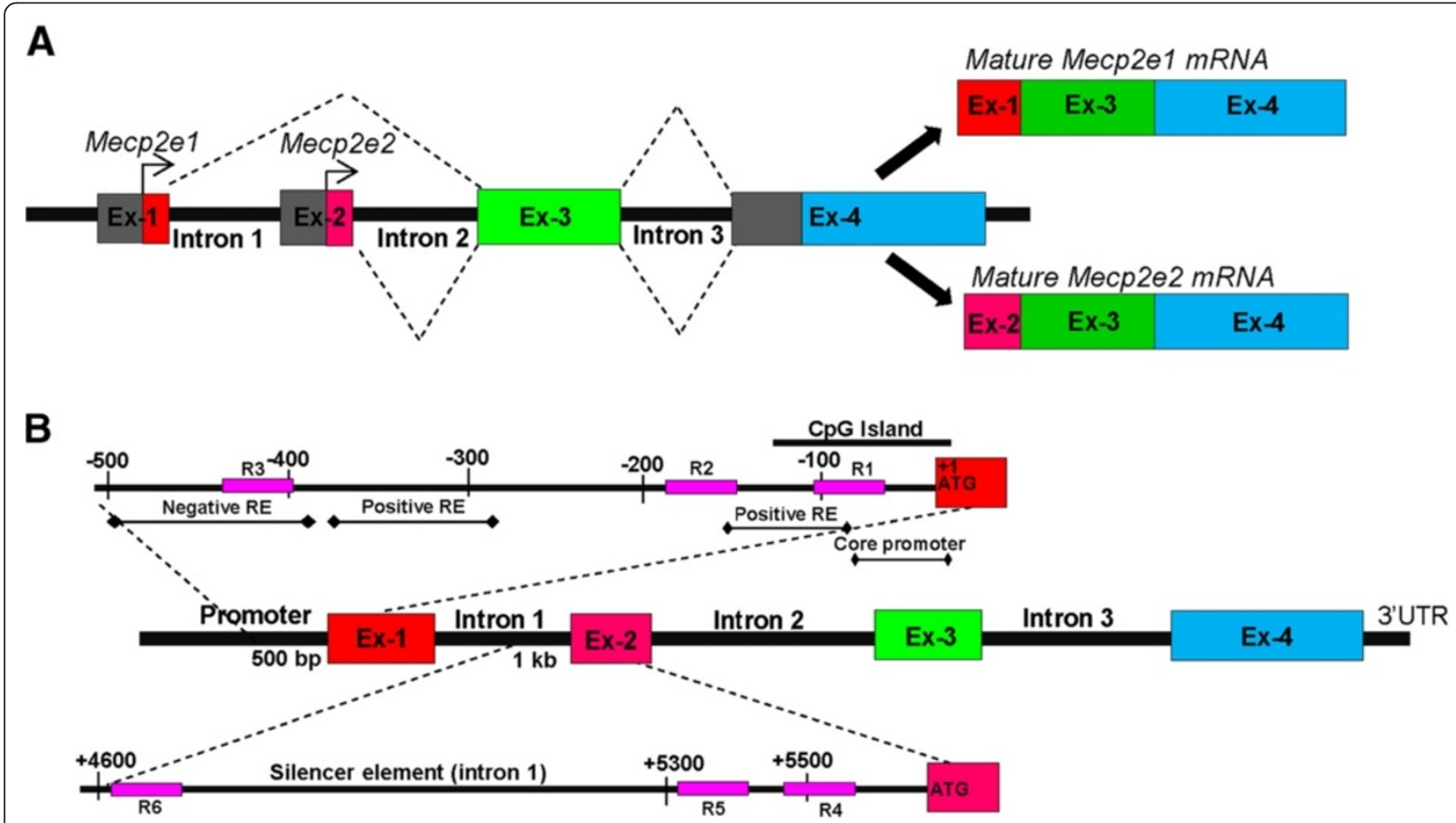

Figure 1 Schematics of the Methyl CpG binding protein 2 gene (Mecp2), Mecp2e1/e2 transcripts, and known regulatory elements (REs).

(A) Generation of MeCP2 isoforms by alternative splicing; mature Mecp2e1 transcripts comprise of exons 1, 3, and 4. Mature Mecp2e2 transcripts comprise of exons 2, 3, and 4 (adapted from [4,11]). Exons are denoted as Ex. (B) Regulatory elements of the MECP2/Mecp2 gene. The MECP2/ Mecp2 gene is reported to be regulated by negative and positive REs within the promoter and a silencer element within the intron 1 (information extracted from [12,13]). For our studies we selected a 500-bp region in the promoter upstream of the exon 1 and a 1-kb region in the intron 1 upstream of the exon 2. Each sequence was divided into three regions, R1 to R3 in the Mecp2 promoter and R4 to R6 in the intron 1. Note that there are no $\mathrm{CpG}$ dinucleotides in the mouse genomic sequence between R5 and R6. 
is generally considered to be an active epigenetic mark $[24,25]$. Promoter methylation is mostly associated with gene silencing [26], while DNA methylation at both intronic and exonic regions are shown to correlate with isoform-specific transcription by alternative splicing or by utilizing alternate promoters $[27,28]$.

Treatment with DNA demethylating agents or DNMT/ Dnmt inhibitors such as decitabine (also called 5-Aza-2' deoxycytidine) is a commonly used method to study the role of DNA methylation in gene expression $[29,30]$. While exposure to decitabine results in DNA demethylation, its subsequent withdrawal causes remethylation or methylation reprogramming [29], providing an excellent platform to uncover the role of DNA methylation in gene expression.

In vitro differentiation of neural precursor cells/neural stem cells (NSC) into different brain cell types is utilized as an acceptable model system to mimic the in vivo neural development [31-36]. Previously, we used a similar in vitro NSC differentiation system to report the first preclinical $M E C P 2$ isoform-specific gene therapy vectors, for future gene therapy applications in Rett syndrome [35]. Further, we introduced differentiating NSC as a suitable in vitro model to study the expression and function of developmentally important genes such as Meis1 in neural development [37]. In the current study, we used this previously characterized system to study the expression and regulation of Mecp2 isoforms during NSC differentiation.

Investigation of $M E C P 2 / \mathrm{MeCP} 2$ expression and function in neurodevelopmental disorders has been the focus of intensive research. However, despite the critical importance of precisely controlled levels of MeCP2 expression in the brain, the underlying regulatory mechanisms have been understudied. Here, we report the correlation between the expression of Mecp2 isoforms and DNA methylation patterns at the Mecp 2 REs at different stages of NSC differentiation. Further, we demonstrate the effect of dynamic changes in DNA methylation induced by exposure and withdrawal of decitabine on the expression of Mecp2/MeCP2 isoforms.

\section{Methods}

\section{Ethics statement}

All experiments were performed in accordance with the standards of the Canadian Council on Animal Care with the approval of the Office of Research Ethics of University of Manitoba. All experimental procedures were reviewed and approved (protocol number 12-031) by the University of Manitoba Bannatyne Campus Protocol Management and Review Committee.

\section{Neural stem cell isolation, culture and differentiation} Embryonic mouse forebrain-derived NSC were isolated from the forebrains of CD-1 mice at embryonic day $(E)$
14.5 and were cultured according to previously described methods [35,37]. Briefly, dissected forebrain tissues were mechanically homogenized in NSC media DMEM/F12 1:1 (Wisent, Quebec, Canada) containing HEPES, glutamine, antibiotic/antimycotic, glucose, recombinant human epidermal growth factor (rhEGF) (Sigma Aldrich, Oakville, Ontario, Canada, $20 \mathrm{ng} / \mathrm{ml}$ ), basic fibroblast growth factor (bFGF) (Upstate (Millipore), Billerica, MA, USA, $20 \mathrm{ng} / \mathrm{ml}$ ), heparin (Sigma Aldrich, Oakville, Ontario, Canada, $2 \mu \mathrm{g} / \mathrm{ml}$ ) and hormone mix. Dissociated single cells were plated at a density of $10^{5}$ cells $/ \mathrm{cm}^{2}$ in NSC media. The media were refreshed every $48 \mathrm{~h}$ and cells were cultured under these conditions for 7 days to generate neurospheres. Primary neurospheres were gently dissociated to single cells by accutase treatment. Dissociated cells were plated on plates coated with growth factor-reduced matrigel (BD Biosciences, Mississauga, Ontario, Canada) at a density of $10^{5}$ cells $/ \mathrm{cm}^{2}$ in DMEM (GIBCO, Life Technologies Inc, Burlington, Ontario, Canada) and 10\% Fetal Bovine Serum (Invitrogen, Life Technologies Inc, Burlington, Ontario, Canada) in the absence of rhEGF and bFGF. Cells were differentiated for 8 days, reported to be sufficient for differentiation of neuronal and glial cells [35,37], and media were changed every other day.

\section{Decitabine treatment}

At the onset of differentiation on day zero (D0), dissociated NSC were treated with $2.5 \mu \mathrm{M}$ decitabine for $48 \mathrm{~h}$. After two days (D2), the media were replaced with fresh media that was refreshed every other day for an extra 6 days (until D8). Control cells were cultured under similar experimental conditions, in the absence of decitabine.

\section{Quantitative measurement of male/female contribution}

Genomic DNA from neural stem cells at D0, D2, D8 and decitabine-treated cells were extracted using the DNeasy Blood and Tissue kit (Qiagen, Ontario, Toronto, Canada), as per manufacturer's instructions. The contribution of male and female sexes were determined by semiquantitative PCR-based amplification of Sry (sex-determining region protein gene in the $\mathrm{Y}$ chromosome) and $\mathrm{Il3}$ (autosomal gene as an internal control) genes, as described previously [38], using the primers listed in Table 1 . The PCR program consisted of an initial denaturation at $95^{\circ} \mathrm{C}$ for 4.5 minutes, followed by 33 cycles of $95^{\circ} \mathrm{C}$ for $35 \mathrm{~s}, 50^{\circ} \mathrm{C}$ for 1 minute, $72^{\circ} \mathrm{C}$ for 1 minute, and a final extension step at $72^{\circ} \mathrm{C}$ for 5 minutes. The amplified products were run on $1.5 \%$ agarose gel and the bands were visualized by ethidium bromide staining. The Sry and Il3 PCR products were identified based on the corresponding sizes (Sry: $402 \mathrm{bp}$, and Il3: $544 \mathrm{bp})$. Intensity of the corresponding bands was quantified using Adobe Photoshop CS5 software. The contribution of either sex was further determined by 
Table 1 List of primers used for PCR

\begin{tabular}{llll}
\hline Gene & Direction & Sequence & Reference \\
\hline Sry & Forward & 5'-TGGGACTGGTGACAATTGTC-3' & [38] \\
& Reverse & 5'-GAGTACAGGTGTGCAGCTCT-3' & \\
II3 & Forward & 5'-GGGACTCCAAGCTTCAATCA-3' & \\
& Reverse & 5'-GGAGGAGGAAGAAAAGCAA-3' & \\
\hline
\end{tabular}

quantitative reverse transcription PCR (qRT-PCR) for Xist (X-inactive specific transcript) gene, as previously described, with minor modifications [39]. The PCR program for Xist included an initial denaturation at $95^{\circ} \mathrm{C}$ for 5 minutes; followed by 35 cycles of $95^{\circ} \mathrm{C}$ for $30 \mathrm{~s}, 53^{\circ} \mathrm{C}$ for $30 \mathrm{~s}, 72^{\circ} \mathrm{C}$ for $30 \mathrm{~s}$, and $78^{\circ} \mathrm{C}$ for $30 \mathrm{~s}$.

Quantitative reverse transcription polymerase chain reaction RNeasy Mini Kit (Qiagen, Ontario, Toronto, Canada) was used for RNA extraction as per the manufacturer's protocol. Preparation of cDNA and qRT-PCR were carried out as described previously [40-42]. Transcript levels of Mecp2 (total), Mecp2e1 [NCBI: NM_001081979.1], Mecp2e2 [NCBI: NM_010788.3], Dnmt genes (Dnmt1, Dnmt3a and Dnmt3b), neuronal genes (Tubulin III (Tub III), NeuN), astrocytic genes (Gfap, S100b), and oligodendrocyte-specific genes (Cnpase, $M b p$ ) were examined by using gene-specific primers (Table 2), as described previously $[37,43]$. The relative expression and fold changes were calculated as described previously [37]. Two-way analysis of variance (ANOVA) and the Student $t$-test were used to calculate significant differences between untreated control and decitabine-treated samples.

\section{Immunofluorescence experiments}

Immunofluorescence (IF) experiments were performed according to previously described protocols [7,35,37]. Primary and secondary antibodies used for IF are listed

Table 2 List of primers used for qRT-PCR

\begin{tabular}{|c|c|c|c|}
\hline Gene & Direction & Sequence & Reference \\
\hline \multirow[t]{2}{*}{ Mecp2 (total) } & Forward & 5'-GGTAAAACCCGTCCGGAAAATG-3' & {$[4]$} \\
\hline & Reverse & 5'-TTCAGTGGCTTGTCTCTGAG-3' & \\
\hline \multirow[t]{2}{*}{ Mecp2e1 } & Forward & 5'-AGGAGAGACTGGAGGAAAAGT-3' & {$[5]$} \\
\hline & Reverse & 5'-CTTAAACTTCAGTGGCTTGTCTCTG-3' & \\
\hline \multirow[t]{2}{*}{ Mecp2e2 } & Forward & 5'-CTCACCAGTTCCTGCTTTGATGT-3' & \\
\hline & Reverse & 5'-CTTAAACTTCAGTGGCTTGTCTCTG-3' & \\
\hline \multirow[t]{2}{*}{ Tubulin III (Tub III) } & Forward & 5'-TCAGCGATGAGCACGGCATA-3' & {$[37]$} \\
\hline & Reverse & 5'-CACTCTTTCCGCACGACATC-3' & \\
\hline \multirow[t]{2}{*}{ Gfap } & Forward & 5'-GCTCACAATACAAGTTGTCC-3' & \\
\hline & Reverse & 5'-ACCTAATTACACAGAGCCAGG-3' & \\
\hline \multirow[t]{2}{*}{ Gapdh } & Forward & 5'-AACGACCCCTTCATTGAC-3' & [43] \\
\hline & Reverse & 5'-TCCACGACATACTCAGCAC-3' & \\
\hline \multirow[t]{2}{*}{ NeuN } & Forward & 5'-GGCAATGGTGGGACTCAAAA-3' & [44] \\
\hline & Reverse & 5'-GGGACCCGCTCCTTCAAC-3' & \\
\hline \multirow[t]{2}{*}{ S100b } & Forward & 5'-GCTGACCACCATGCCCCTGTAG-3' & [45] \\
\hline & Reverse & 5'-CTGGCCATTCCCTCCTCTGTC-3' & \\
\hline \multirow[t]{2}{*}{ Mbp } & Forward & 5'-GGCACGCTTTCCAAAATCT-3' & {$[46]$} \\
\hline & Reverse & 5'-CCATGGGAGATCCAGAGC-3' & \\
\hline \multirow[t]{2}{*}{ Cnpase } & Forward & 5'-CATCCTCAGGAGCAAAGGAG-3' & {$[47]$} \\
\hline & Reverse & 5'-TGAATAGCGTCTTGCACTCG-3' & \\
\hline \multirow[t]{2}{*}{ Dnmt1 } & Forward & 5'-AGGGAAAAGGGAAGGGCAAG-3' & {$[48]$} \\
\hline & Reverse & 5'-AGAAAACACATCCAGGGTCCG-3' & \\
\hline \multirow[t]{2}{*}{ Dnmt3a } & Forward & 5'-CAGCGTCACACAGAAGCATATCC-3' & \\
\hline & Reverse & 5'-GGTCCTCACTTTGCTGAACTTGG-3' & \\
\hline \multirow[t]{2}{*}{ Dnmt3b } & Forward & 5'-CCTGCTGAATTACTCACGCCCC-3' & \\
\hline & Reverse & 5'-GTCTGTGTAGTGCACAGGAAAA-3' & \\
\hline \multirow[t]{2}{*}{ Xist } & Forward & 5'-TTGTGGCTTGCTAATAAT-3' & [39] \\
\hline & Reverse & 5'-AAACCCCATCCTTTATG-3' & \\
\hline
\end{tabular}


in the Tables 3 and 4, respectively. Immunofluorescence signals were detected by an Axio Observer $\mathrm{Z} 1$ inverted microscope and LSM710 Confocal microscope from Carl Zeiss. Images were obtained with AxioVision 4.8 (Carl Zeiss Canada Ltd. Ontario, Toronto, Canada) and Zen 2009 software and assembled using Adobe Photoshop CS5 and Adobe Illustrator CS5. For quantification analysis in neurospheres, three neurospheres were randomly selected and all of the cells within each neurosphere were counted based on 4',6-diamidino-2-phenylindole (DAPI) staining. For cell quantification of differentiating cells at D2 and D8, 8 to 10 random fields were selected under the microscope. Approximately 250 cells from the D2 population and 750 cells from the D8 population were counted based on DAPI labeling. The cell counting was done using the ImageJ program.

\section{Nuclear extractions and western blotting}

Nuclear extraction from D2 and D8 NSC were carried out using the NE-PER Nuclear and Cytoplasmic Extraction Kit (Thermo Scientific, Ontario, Toronto, Canada), as per the manufacturer's instructions. Western blot (WB) experiments were conducted according to previously described protocols [49-51], and quantification of the signals was performed as reported [7]. ACTIN or glyceraldehyde-3-phosphate dehydrogenase (GAPDH) was used as a loading control. Student $t$-test was used to determine statistical significance between control and treated cells. Primary and secondary antibodies used for WB are listed in the Tables 3 and 4, respectively.

\section{DNA dot blot assay for $5 \mathrm{mC}$ and $5 \mathrm{hmC}$}

Genomic DNA was isolated using the DNeasy Blood and Tissue kit (Qiagen, Ontario, Toronto, Canada). DNA dot blot was performed using a previously described protocol [52], with minor modifications. The DNA blotted membranes were probed with either $5 \mathrm{mC}$ or $5 \mathrm{hmC}$ antibody (Tables 3 and 4). Total DNA levels were detected by staining the same membrane with $0.02 \%$ methylene blue (MB) in $0.3 \mu \mathrm{M}$ sodium acetate ( $\mathrm{pH} 5.2$ ). Adobe Photoshop CS5 software was used to quantify dot blot signals.

\section{Bisulfite pyrosequencing}

Genomic DNA was isolated as described in the previous section. Primer design and bisulfite pyrosequencing experiments were conducted as a service by The Hospital for Sick Children (SickKids), Toronto, Canada, as reported elsewhere [53], using the primers listed in Table 5. The regions analyzed for the methylation patterns are shown in Figure 1B. Specific CpG dinucleotides that are analyzed within each region are shown in Additional file 1.

\section{Correlation analysis}

The correlation between DNA methylation at the Mecp2 REs and expression of Mecp2 isoforms was determined using the Pearson's correlation analysis and linear regression. The Pearson's correlation coefficient $(r)$ was calculated for average methylation against each Mecp2 isoform, over entire regions and for individual $\mathrm{CpG}$ sites within each region. The strength of correlation was considered as follows: weak, $0<r<0.3$; moderate, $0.3<r<0.4$; strong,

Table 3 Primary antibodies used

\begin{tabular}{|c|c|c|c|}
\hline Primary antibody & Application and dilution & Description & Source \\
\hline MeCP2 (C-terminal) & IF 1:200 & Rabbit polyclonal & Millipore, Billerica, MA, USA, 07-013 \\
\hline \multirow[t]{2}{*}{ MeCP2 (C-terminal) } & WB 1:100 & Mouse monoclonal & Abcam, Ontario, Toronto, Canada, Ab50005 \\
\hline & IF 1:200 & & \\
\hline MeCP2E1 & WB 1:100 & Chicken polyclonal & Custom-made [7] \\
\hline GFAP & IF 1:200 & Mouse monoclonal & Invitrogen, Life Technologies Inc, Burlington, Ontario, Canada 421262 \\
\hline TUBULIN III (TUB III) & IF 1:200 & Mouse monoclonal & Chemicon, Millipore, Billerica, MA, USA MAB1637 \\
\hline OLIG2 & IF 1:200 & Rabbit polyclonal & Millipore, Millipore, Billerica, MA, USA, AB9610 \\
\hline NESTIN & IF $1: 230$ & Rat monoclonal & Developmental Studies Hybridoma Bank, Rat-401c \\
\hline $\mathrm{S} 100 \mathrm{~B}$ & IF 1:100 & Mouse monoclonal & Abcam, Ontario, Toronto, Canada, ab4066 \\
\hline CNPase & IF 1:100 & Mouse monoclonal & Covance, SMI-91R \\
\hline MBP & IF 1:100 & Rabbit polyclonal & Abcam, Ontario, Toronto, Canada, ab40390 \\
\hline NEUN & IF 1:200 & Mouse monoclonal & Millipore, Billerica, MA, USA, Mab377 \\
\hline $\mathrm{K} 167$ & IF 1:200 & Rabbit polyclonal & Santa cruz, Dallas, Texas, USA, sc-15402 \\
\hline \multirow[t]{2}{*}{$5 \mathrm{mC}$} & Dot blot 1:1,000 & Mouse monoclonal & Abcam, Ontario, Toronto, Canada, Ab73938 \\
\hline & IF 1:200 & & \\
\hline $5 \mathrm{hmC}$ & Dot blot 1:1,000 & Rabbit polyclonal & Active Motif, 39769 \\
\hline
\end{tabular}

MeCP2, Methyl CpG binding protein; GFAP, Glial fibrillary acidic protein; OLIG2, Oligodendrocyte lineage transcription factor 2; CNPase, 2',3'-Cyclic-nucleotide 3'-phosphodiesterase, MBP, Myelin basic protein; NEUN, NEUronal Nuclei; 5mC, 5-methylcytosine; 5hmc, 5-hydroxymethylcytosine; IF, Immunofluorescence; WB, Western blot. 
Table 4 Secondary antibodies used

\begin{tabular}{lll}
\hline Secondary antibody & Application and dilution & Source \\
\hline FITC conjugated goat anti rabbit IgG & IF 1:400 & Jackson Immunoresearch, PA, USA, 111-095-144 \\
Rhodamine Red-X conjugated goat anti mouse IgG & IF 1:400 & Jackson Immunoresearch, PA, USA, 115-259-146 \\
Dylight 649 conjugated goat anti chicken IgY & IF 1:400 & Jackson Immunoresearch, PA, USA, 103-485-155 \\
Dylight 649 conjugated donkey anti goat IgG & IF 1:400 & Jackson Immunoresearch, PA, USA, 705-494-147 \\
Alexa Fluor 594 conjugated donkey anti mouse IgG & IF 1:1,000 & Life Technologies Inc, Ontario, Canada, 987237 \\
Alexa Fluor 448 conjugated donkey anti rabbit IgG & IF 1:1,000 & Life Technologies Inc, Ontario, Canada, 913921 \\
Peroxidase-Affinipure Gt anti-mouse IgG & WB 1:7,500 & Jackson ImmunoResearch, PA, USA, 115-035-174 \\
& Dot blot 1:7,500 & \\
\hline
\end{tabular}

IF, Immunofluorescence; WB, Western blot.

$0.4<r<0.7$; or very strong, $0.7<r<1.0$. A negative $r$-value indicates an inverse/negative correlation whereas a positive $r$-value indicates direct/positive correlation. Statistical significance was determined at $P<0.05$.

\section{Results}

Dynamic expression of Mecp2 isoforms during NSC differentiation and DNA methylation patterns at the Mecp2 regulatory elements

Primary neural stem cells were isolated from the embryonic forebrain at E14.5 and were cultured in the presence of growth factors to generate primary neurospheres. After 7 days in culture, primary neurospheres were dissociated and cultured under differentiation conditions for 8 days, reported to be sufficient for differentiation of both neuronal and glial cells $[35,37]$ (Figure 2A). In agreement with our previous report [37] the proliferating primary neurospheres expressed NESTIN and KI67 (Figure 2B). Similar to our previous reports [35,37] differentiated NSC at D8 consisted of a mixed population of neurons, astrocytes, and oligodendrocytes (Figure 2C). The composition of the D8 population was determined by detection of cell typespecific markers (TUB III $(4.7 \% \pm 0.8$ mean \pm standard error of the mean (SEM)), Glial fibrillary acidic protein (GFAP) $(54.4 \% \pm 1.1)$, S100B $(15.9 \% \pm 2.7), 2$ ',3'-Cyclic-nucleotide 3 '-phosphodiesterase (CNPase) $(1.6 \% \pm 0.2)$, Myelin basic protein $(\mathrm{MBP})(2.6 \% \pm 0.42)$, Oligodendrocyte lineage transcription factor 2 (OLIG2) $(36.2 \% \pm 1.8)$ and KI67 $(92.4 \% \pm 1.3)$ ) by immunofluorescence (Figure 2C). KI67 is not a cell type-specific marker, but rather reflects the fraction of cycling cells within differentiating NSC. Indicating that the cells at an early stage of differentiation

Table 5 Primers used in bisulfite pyrosequencing

\begin{tabular}{|c|c|}
\hline Mecp2 region & Sequence \\
\hline \multirow[t]{3}{*}{ Region 1} & F: 5'-TGGGTTITATAATTAATGAAGGGTAA-3' \\
\hline & R: 5'-CGCCAGGGTTTTCCCAGTCACGACATTTTACCACAACCCTCTCT-3' \\
\hline & S: 5'-AGGTGTAGTAGTATATAGG-3' \\
\hline \multirow[t]{3}{*}{ Region 2} & F: 5'-AGTTTGGGTTTATAATTAATGAAGGG-3' \\
\hline & R: 5'-CGCCAGGGTTTTCCCAGTCACGACATTTTACCACAACCCTCTCT-3' \\
\hline & S: 5'-AAGGGTAATTTAGATAAAGAGTAAG-3' \\
\hline \multirow[t]{3}{*}{ Region 3} & F: 5'-GGTGAATTATTAAGTAGGGAGGTTTTAA -3' \\
\hline & R: 5'-CGCCAGGGTTTCCCAGTCACGACAAAAAAAAAACCAACCCCATTCAACTAC -3' \\
\hline & S: 5'-AGTAGGGAGGTITTAATAG -3' \\
\hline \multirow[t]{3}{*}{ Region 4} & F: 5'-GTITTAAAAAGTTITGGGAAAAGGTGTAGT -3' \\
\hline & R: 5'-CGCCAGGGTITTCCCAGTCACGACCTAAACCCTAACATCCCAACTACCAT-3' \\
\hline & S: 5'-AGTTTAATGGGGATTITTAATT -3' \\
\hline \multirow[t]{3}{*}{ Region 5} & F: 5'-AGTAGAAGTTATTATTTGTGGTGTGTAT -3' \\
\hline & R: 5'-CGCCAGGGTTTTCCCAGTCACGACACTATATTACTTCCCAACTCAACTAATT -3' \\
\hline & S: 5'-AGAGGTGTAAGGATTTT -3' \\
\hline \multirow[t]{3}{*}{ Region 6} & F: 5'-GAAGTAGGAAGAATTGAGTTTGAGGATAG -3' \\
\hline & R: 5'-CGCCAGGGTTITCCCAGTCACGACATCTATACACTACCCACATATAATACC -3' \\
\hline & S: 5'-GTTTGAGGATAGTTTGAAT -3' \\
\hline
\end{tabular}

F: Forward PCR primer, R: Reverse PCR primer (Biotinylated), S: Sequencing primer. 


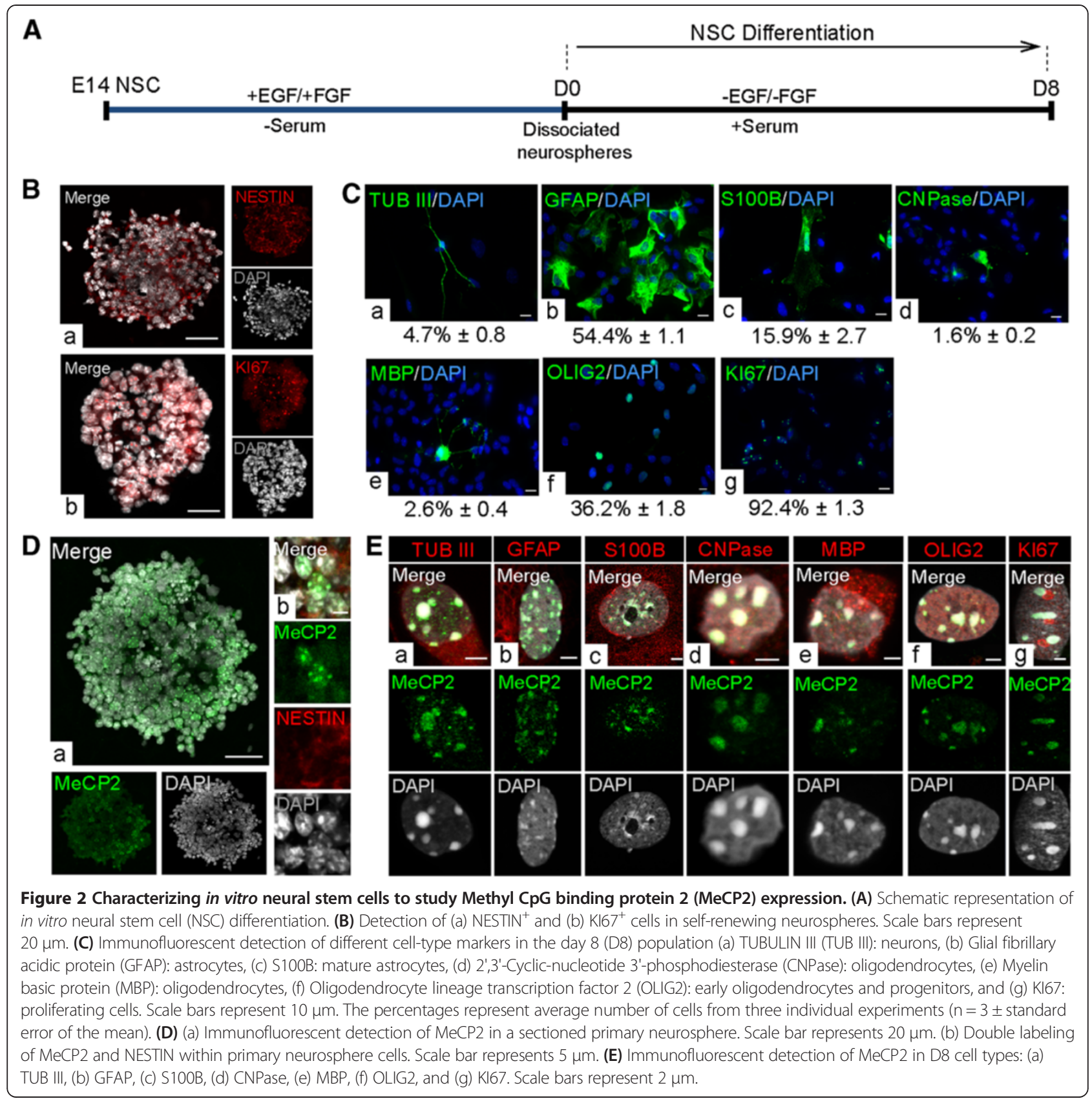

are actively dividing, expression of KI67 $(98.8 \% \pm 0.8)$ was also detected in D2 cells (Additional file 2). Our detection of KI67 in the majority of cells at D8 indicates that although most differentiating NSC are actively dividing, fewer than $10 \%$ of cells are post-mitotic and may include neurons (TUB $\mathrm{III}^{+}$) or non-proliferating cells committed towards neuronal cell fate. Taken together, this in vitro NSC differentiation system provided a suitable model system consisting of the three main neural cell types (neurons, astrocytes and oligodendrocytes) in the brain to study Mecp2/MeCP2 isoforms during neural differentiation.
First, we confirmed MeCP2 expression in neurospheres at D0 and differentiated NSC at D8 by IF studies. We used an antibody that was raised against the MeCP2 Cterminus and recognizes both isoforms. Characteristic punctate nuclear expression of $\mathrm{MeCP} 2$ was detected in $41 \%$ of neurosphere cells (Figure 2D, a), which were positive for the NSC marker NESTIN (Figure 2D, b). At D8 of NSC differentiation, MeCP2 protein was detected in all cell types in the D8 differentiated progenies, including neurons, astrocytes, and oligodendrocytes (Figure 2E). Indicating the expression of $\mathrm{MeCP} 2$ in proliferating cells, 
we detected $\mathrm{MeCP} 2$ in $\mathrm{KI}_{6} 7^{+}$cells in the $\mathrm{D} 8$ population (Figure 2E, g). The detected nuclear MeCP2 signals were enriched at the heterochromatin-rich regions of all three cell types. These observations are consistent with our previous reports on MeCP2 nuclear expression in in vivo differentiated primary neurons and astrocytes [7].

Next, we investigated Mecp 2 isoform-specific transcript expression at three stages of NSC differentiation: undifferentiated cells (D0), cells at an early stage of differentiation (D2), and cells at a later stage of differentiation (D8). Distinct and mirror-like (reciprocal) transcript expression patterns for Mecp 2 isoforms were observed during NSC differentiation (D0, D2, and D8) (Figure 3A), suggesting possible differential regulation of these isoforms during NSC differentiation. Expression of Mecp2e1 was reduced from D0 to D2 $(2.7$-fold, $P<0.001)$ and was slightly elevated from D2 to D8. In contrast, Mecp2e2 expression increased from D0 to D2 (3.1-fold, $P<0.05)$, but declined from D2 to D8 (4.2-fold, $P<0.01)$. At each of these studied differentiation stages, the expression ratio between the two isoforms (Mecp2e1/Mecp2e2) varied significantly (D0, 5.99; D2, 0.69; D8, 4.62) (Additional file 3). At D0 and D8, Mecp2e1 expression was significantly higher than Mecp2e2 (D0, $P<0.01$, and D8, $P<0.05$ ). In contrast at D2, Mecp2e2 expression was significantly higher than Mecp2e1 (D2, $P<0.05$ ). These observations imply differential regulation of Mecp 2 isoforms and possible changes in alternative splicing of Mecp2 at different stages of NSC differentiation.

$M E C P 2 / M e c p 2$ expression is known to be regulated by REs found within its promoter and intron 1 . The altered $\mathrm{MeCP} 2$ expression in autistic patients and in mouse brains subjected to stress is correlated with increased MECP2/Mecp2 promoter methylation [2,12,13,18-20]. Therefore, we hypothesized that DNA methylation at the Mecp 2 promoter and intron 1 might impact Mecp2 isoform-specific expression. For DNA methylation analysis by bisulfite pyrosequencing, we selected three regions within the Mecp 2 promoter (named R1 to R3, hereafter) and three regions within the intron 1 (named R4 to R6, hereafter) (Figure 1B). These regions harbored different numbers of $\mathrm{CpG}$ sites; promoter regions $\mathrm{R} 1$ : $\mathrm{CpG}$ island contains 13 CpG sites; R2: 4 CpG sites; R3: 2 CpG sites, and intron 1 regions R4: 1 CpG site; R5: 1 CpG site; and R6: 2 CpG sites.

Pyrosequencing analysis of R1-R6 indicated that downregulation of Mecp2e1 and upregulation of Mecp2e2 from D0 to D2 were associated with slight, but significant demethylation of Mecp 2 promoter R1 (2.3\%, $P<0.05)$. Similarly, upregulation of Mecp2e1 and downregulation of Mecp2e2 from D2 to D8 were associated with hypermethylation of Mecp2 intron 1 R5 $(2.4 \%, P<0.01)$ (Figure 3B). Detected expression changes in Mecp2 isoforms from D0 to D8 were associated with demethylation of Mecp2 promoter R1 (2.6\%, $P<0.05)$, and hypermethylation of Mecp 2 intron $1 \mathrm{R} 4$ (4.6\%, $P<0.05)$. In all cases, the differences in average percentage methylation between D0, D2 and D8 were relatively small, but statistically significant and ranging between 2 to $5 \%$. Previous reports have shown that an increase in the overall $M E C P 2$ promoter methylation by approximately 2.0 to $2.5 \%$ in male autistic patients correlates with significantly reduced $M E C P 2$ expression levels [2]. In mouse brain exposed to maternal separation and stress, DNA methylation changes that are as little as 2 to
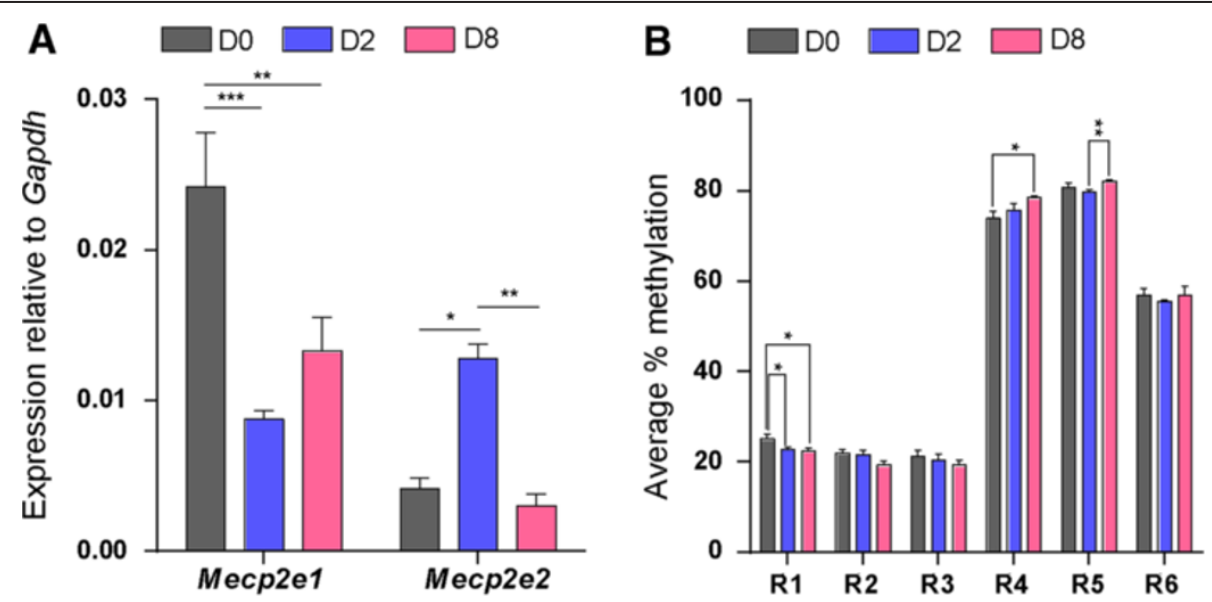

Figure 3 Methyl CpG binding protein 2 gene (Mecp2) isoform-specific transcript expression and DNA methylation at the Mecp2 regulatory elements during neural stem cell (NSC) differentiation. (A) Analysis of Mecp2e1 and Mecp2e2 transcript levels during NSC differentiation: $\mathrm{n}=3 \pm$ standard error of the mean (SEM). Significant differences: ${ }^{* * * *} P<0.001 ;{ }^{* * *} P<0.01 ;{ }^{*} P<0.05$. (B) Average percentage methylation over Mecp2 promoter and intron 1 regions at day 0 (D0), day 2 (D2) and day 8 (D8) during NSC differentiation. The regions are promoter regions R1, CpG island contains 13 CpG sites; R2, 4 CpG sites; R3, 2 CpG sites, and intron 1 regions R4, 1 CpG site; R5, 1 CpG site; and R6, 2 CpG sites; $n=3 \pm$ SEM. Significant differences: ${ }^{* *} P<0.01 ;{ }^{*} P<0.05$. Gapdh, glyceraldehyde-3-phosphate dehydrogenase gene. 
$5 \%$ at individual $\mathrm{CpG}$ sites of the $M e c p 2$ promoter are associated with significantly reduced MeCP2 expression [20]. As even slightly altered $M E C P 2 / M e c p 2$ promoter methylation (2 to 5\%) affects $M E C P / M e c p 2$ gene expression in the human and mouse brain, it is likely that the statistically significant changes detected in the present study might be biologically important for Mecp $2 e 1$ and/or Mecp $2 e 2$ expression.

As mentioned, the ratio of Mecp 2 splice variants was changed at different stages of NSC differentiation. Therefore, we performed Pearson's correlation analysis between Mecp2e1/Mecp2e2 expression ratio and DNA methylation at R1, R4 and R5 (the three regions that showed significant changes) during NSC differentiation. Pearson's correlation coefficient $(r)$ represents the strength of correlation, with negative $r$ indicating inverse correlation, and positive $r$ indicating direct correlation between DNA methylation and the Mecp2e1/Mecp2e2 splice ratio. We detected a statistically significant positive correlation $(r>0.9, P<0.01)$ between Mecp2e1/Mecp2e2 splice ratio at D2 and DNA methylation at intron 1 R4 (Additional file 3). Although it is possible that intron 1 (R4) may play a role in alternative splicing of $M e c p 2$, further investigations are required to establish the involvement of DNA methylation in $M e c p 2$ transcriptional splicing.

As $M E C P 2 / M e c p 2$ is an $\mathrm{X}$-linked gene, it is possible that the observed changes in Mecp 2 expression are due to a shift in the number of cells derived from male and female embryos. To exclude such a possibility, we determined the contribution from the male/female embryonic cells during NSC differentiation using a semiquantitative PCR-based method. Genomic DNA was extracted from each differentiation stage and subjected to PCR analysis for the presence of the Sry gene found in the Y chromosome. The autosomal gene Il3 was used an internal control. The adult male brain cortex was used as a positive control for the presence of the male genomic DNA (Additional file 4: Figure S4A). We did not observe statistically significant changes in the ratio of Sry/Il3 in the cells collected at different stages of differentiation, indicating that the ratio of male/female differentiating NSC were unchanged (Additional file 4: Figure S4B). To further confirm the contribution from the male/female gender, we tested the transcript levels of Xist gene (the gene is involved in X-chromosome inactivation) by qRT-PCR. We did not detect any significant change in Xist gene expression at different stages of NSC differentiation (Additional file 4: Figure S4C). These results indicate that our observed changes in Mecp 2 expression are not due to altered contribution of male and female cells. Taken together, our results suggest a possible link between the Mecp2 isoform-specific expression and DNA methylation at the Mecp2 REs within the Mecp2 promoter and intron 1 during NSC differentiation.

\section{Decitabine exposure leads to Mecp2e1 upregulation but its withdrawal downregulates both Mecp2 isoforms to different extents}

To further study the impact of DNA demethylation/ remethylation in Mecp2 isoform-specific expression, we treated dissociated neurosphere cells with $2.5 \mu \mathrm{M}$ decitabine for $48 \mathrm{~h}$, at the onset of NSC differentiation (D0) (Figure 4A). At D2, decitabine was withdrawn from the media and cells were kept in culture for another 6 days until D8, to study the effect of DNA remethylation (Figure 4A). First, as a proof of principle, we verified whether decitabine acted as a DNA demethylating agent in our system. Global change in DNA methylation was determined by IF for $5 \mathrm{mC}$ and DNA dot blot assay for both $5 \mathrm{mC}$ and $5 \mathrm{hmC}$. As expected, IF experiments showed that $5 \mathrm{mC}$ nuclear signals were noticeably lower in decitabine-treated NSC compared to D2 control untreated cells (Figure 4B). DNA dot blot assays indicated that decitabine treatment resulted in reduced $5 \mathrm{mC}$ levels (3.79fold, $P<0.05$ ), with slight but statistically insignificant increase in $5 \mathrm{hmC}$ levels (Figure $4 \mathrm{C}$-D). In contrast, decitabine withdrawal led to re-establishment of global DNA methylation $(5 \mathrm{mC})$ at D8 as detected by IF (Figure 4F). Furthermore, DNA dot blot assay showed DNA methylation reprogramming upon decitabine withdrawal, with elevated $5 \mathrm{mC}$ levels $(1.5$-fold, $P<0.05)$, and relatively unchanged $5 \mathrm{hmC}$ levels in decitabine-treated NSC compared to controls (Figure 4G-H). Although globally altered $5 \mathrm{mC}$ levels were expected following decitabine treatment in agreement with previous studies $[54,55]$, the observed effect of decitabine to slightly increase $5 \mathrm{hmC}$ levels was novel and might be biologically important.

DNA demethylating agents can function as cytosine analogues and/or as Dnmt/DNMT inhibitors [56]. Therefore, we investigated Dnmt expression levels in decitabinetreated differentiating NSC by qRT-PCR. In accordance with reduced DNA methylation levels at D2, decitabine treatment caused significant inhibition of transcript levels of all three DNA methyltransferases (Dnmt1, 1.7-fold, $P<0.05$; Dnmt3a, 1.5-fold, $P<0.05$ and Dnmt3b, 2.5-fold, $P<0.01$ ) (Figure 4E). Even though we anticipated that decitabine withdrawal would restore Dnmt levels, only Dnmt1 levels were elevated (2.2-fold, $P<0.05)$, whereas both Dnmt3a (1.4-fold, $P<0.05)$ and Dnmt3b (1.8-fold, $P=0.06$ ) levels remained inhibited (Figure 4I). In summary, these results indicate that decitabine functions as a DNA demethylating agent in differentiating NSC and globally affects DNA methyl marks. Additionally, our data indicate that decitabine withdrawal would lead to DNA methylation reprogramming in differentiating NSC.

Next, we investigated possible changes in Mecp2/ MeCP2 expression induced by decitabine. Quantitative RT-PCR experiments indicated that decitabine treatment at D2 caused slight but statistically significant upregulation 


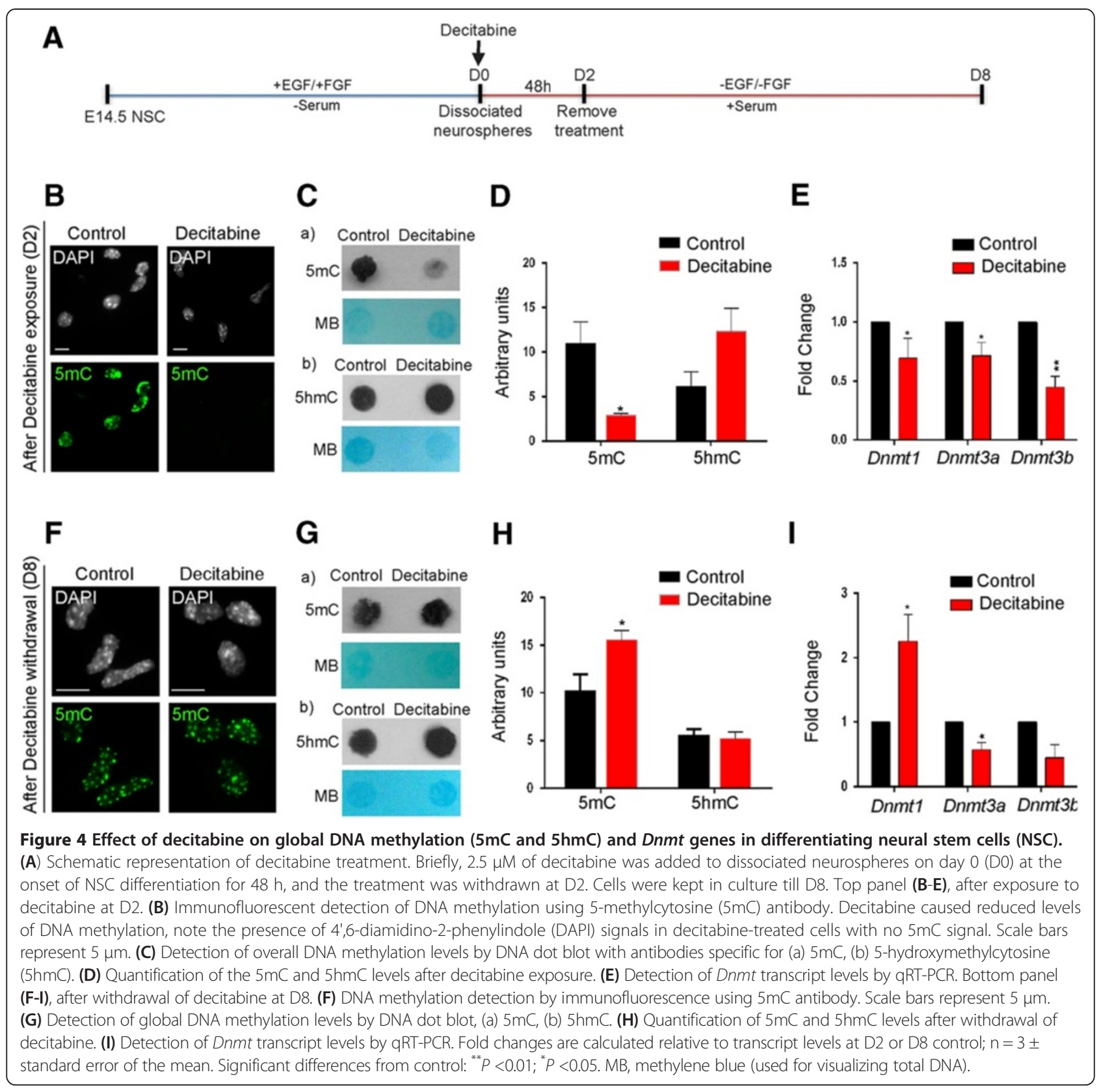

of Mecp2e1 (1.41-fold, $P<0.05)$, with minimal and insignificant increased levels of the total Mecp2 (1.2-fold, $P=$ 0.5 ), and unchanged levels of Mecp2e2 (Figure 5A). Analysis of protein levels by WB showed that decitabine upregulated total MeCP2 (2.5-fold, $P<0.05)$, and MeCP2E1 (3.1-fold, $P<0.05$ ) protein expression (Figure 5B-C). However, the lack of an antibody specific for MeCP2E2 limited our investigation of MeCP2E2 protein levels. Correlation analysis of transcript and protein levels of Mecp $2 / \mathrm{MeCP} 2$ at D2 indicated significant correlation between the detected transcript and protein expression (Mecp2/MeCP2 $(r=0.97, P<0.05)$ and Mecp2e1/MeCP2E1 $(r=0.98, P<0.01)$; Figure 5G).
In contrast to D2, withdrawal of decitabine at D8 significantly downregulated the transcript expression levels of Mecp2e1 (1.92-fold, $P<0.001)$, Mecp2e2 (1.39-fold, $P<0.05)$ and the total Mecp2 (1.52-fold, $P<0.01)$ (Figure 5D). Similar to the transcript levels, decitabine withdrawal resulted in downregulation of total MeCP2 (3.2-fold, $P<0.001$ ), and MeCP2E1 (4.3-fold, $P<0.05)$ protein expression levels (Figure 5E-F). A similar correlation analysis between transcript and protein levels of Mecp2/MeCP2 at D8 did not show any statistically significant correlation (Mecp2/MeCP2 $(r=0.6, P=0.2)$, and Mecp2e1/MeCP2E1 ( $r=0.6, P=0.3)$; Figure $5 \mathrm{H})$. These observations emphasize that even minor change in 

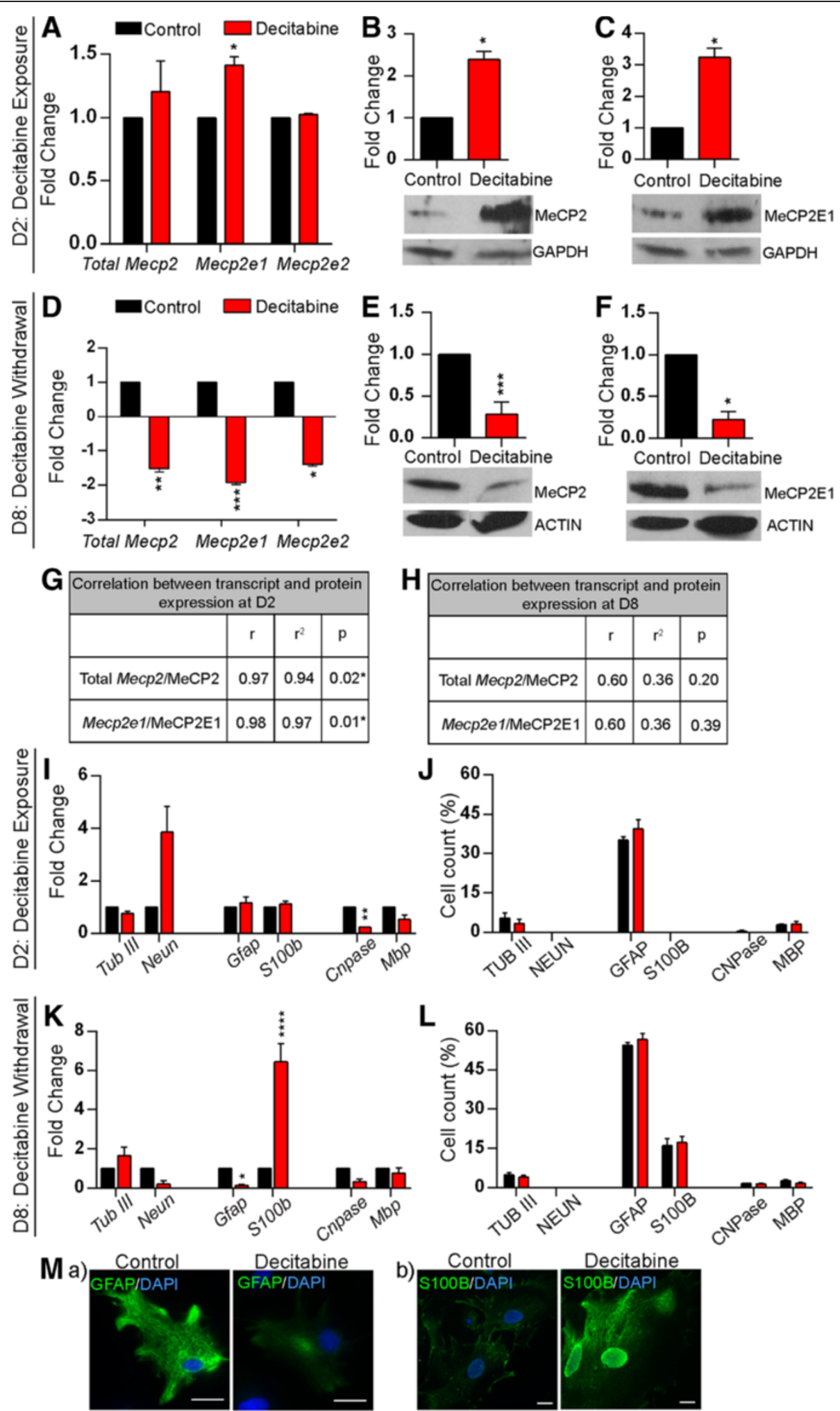

L
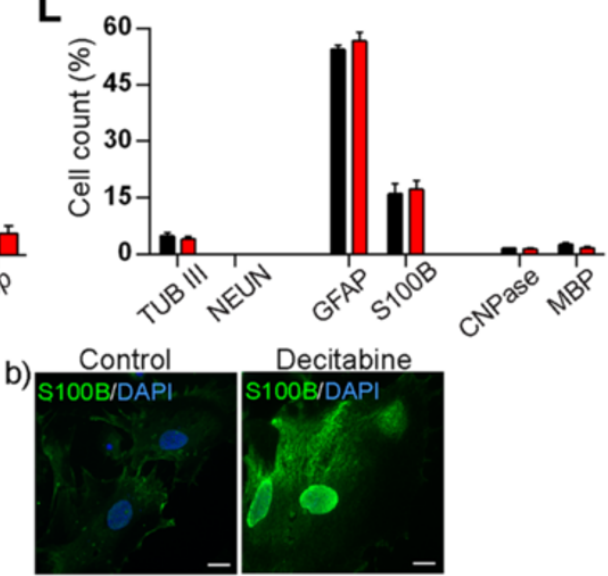
(See figure on previous page.)

Figure 5 Effect of decitabine exposure and withdrawal on Methyl CpG binding protein gene (Mecp2/MeCP2) expression. (A-C), After exposure to decitabine at D2. (A) Analysis of Mecp2 (total), Mecp2e1 and Mecp2e2 transcript levels by qRT-PCR. (B) Detection of MeCP2 (total) protein expression levels by western blot; $n=2 \pm$ standard error of the mean (SEM). (C) Detection of MeCP2E1 protein expression levels by western blot; $n=2 \pm$ SEM. (D-F), After withdrawal of decitabine at day 8 (D8). (D) Analysis of total Mecp2, Mecp2e1 and Mecp2e2 transcript levels by qRT-PCR. (E) Detection of MeCP2 (total) protein expression levels by western blot. (F) Detection of MeCP2E1 protein expression levels by western blot. (G-H) Pearson's correlation analysis of the relation between Mecp2 transcript levels and MeCP2 protein levels at D2 (G) and D8 (H); $r=$ Pearson's correlation coefficient, $r^{2}=$ coefficient of determination. (I) Transcript detection of cell type-specific markers for neurons (Tub III, NeuN); astrocytes (Gfap, S100b); oligodendrocytes (Cnpase, Mbp) by qRT-PCR in D2 control and decitabine-treated cells. (J) Quantification of neurons, astrocytes and oligodendrocytes using cell type-specific markers by immunofluorescence in D2 control and decitabine-treated cells. (K) Transcript detection of cell type-specific markers for neurons (Tub III, NeuN); astrocytes (Gfap, S100b); oligodendrocytes (Cnpase, Mbp) by qRT-PCR in D8 control and decitabine-treated cells. (L) Quantification of neurons, astrocytes and oligodendrocytes using cell type-specific markers in D8 control and decitabine-treated cells. (M) Comparison of immunofluorescent detection of (a) Glial fibrillary acidic protein (GFAP) and (b) S100B between control and decitabine-treated cells. Images were taken at the same exposure time. Scale bars represent 20 um. For all the panels, fold changes are calculated relative to expression levels at D2 or D8 controls. Significant differences from controls: ${ }^{* * * *} P<0.0001 ;{ }^{* * *} P<0.001 ;{ }^{* *} P<0.01$; ${ }^{*} \mathrm{P}<0.05, \mathrm{n}=3 \pm \mathrm{SEM}$, unless specifically mentioned.

Mecp2 transcript levels are biologically important and can result in significantly altered $\mathrm{MeCP} 2$ protein expression levels.

Next, we aimed to study whether the detected changes in $M e c p 2 / \mathrm{MeCP} 2$ expression were due to changes in cell population in response to decitabine treatment. Therefore, we studied the effect of decitabine on cell fate commitment of differentiating NSC at D2 and D8. After decitabine exposure at D2, we examined the expression of cell type-specific markers (neurons: Tub III, NeuN; astrocytes: Gfap, S100b; oligodendrocytes: Cnpase, Mbp) at the transcript levels by qRT-PCR. Comparing the control and decitabine-treated cells, we did not detect any statistically significant change in these cell type-specific genes, except for significant downregulation of Cnpase (9-fold, $P<0.01$ ) (Figure 5I). In order to determine whether any of these detected changes in transcript levels are represented in the number of cells expressing each corresponding cell type-specific marker, we performed IF experiments with specific antibodies against these markers (Figure 5J). IF experiments showed that there was no significant change in the number of TUB $\mathrm{III}^{+}, \mathrm{GFAP}^{+}, \mathrm{CNPase}^{+}$, or $\mathrm{MBP}^{+}$cells. However, we did not find any $\mathrm{NEUN}^{+}$, or $\mathrm{S}_{100 \mathrm{~B}^{+}}$cells in the control or decitabine-treated populations at D2, probably because these cells are still in the early stages of differentiation (Figure 5J). In the D8 population, decitabine treatment led to insignificant changes in the transcript levels for all neuronal and oligodendrocyte markers compared to control untreated cells. Additionally, Gfap expression in decitabine-treated cells was downregulated 5.5-fold, whereas $S 100$ b expression was upregulated to a similar extent (6-fold) (Figure 5K). Quantification of differentiated neurons, astrocytes and oligodendrocytes at D8 by IF did not show any significant change in the cell-fate commitment of these cells (Figure 5L). However, reduced Gfap expression in decitabine-treated cells without any changes in the number of $\mathrm{GFAP}^{+}$cells might be explained by the reduced intensity of GFAP staining relative to the control astrocytes, since the images were taken at the same exposure level (Figure 5M, a). Similarly, the significant upregulation of $S 100 \mathrm{~b}$ transcript levels by decitabine with no change in the number of $\mathrm{S}_{100 \mathrm{~B}}{ }^{+}$cells could be explained by the increased intensity of S100B in decitabine-treated cells, when the images were taken at the same exposure time (Figure 5M, b). Taken together, these results suggest that decitabine has minimal effect on the differentiated number of neurons, astrocytes and oligodendrocytes under the described conditions. They further suggest that the detected changes in Mecp 2 expression are not likely due to altered population of differentiating cell types.

Next, we investigated whether the observed altered Mecp2/MeCP2 expression was due to changes in the number of cells deriving from male and female embryos. Detection of Sry and Il3 by PCR indicated that the ratio of Sry/Il3 was relatively similar in D2 control and decitabine-treated populations (Additional file 4: Figure S4D). Similar PCR analysis at D8 also showed no significant differences in the ratio of Sry/Il3 between D8 control and decitabine-treated cells (Additional file 4: Figure S4E). Furthermore, qRT-PCR analysis of the Xist gene expression in both D2 and D8 populations with and without decitabine treatment showed no significant change in Xist transcript expression levels between the control and decitabine-treated cells (Additional file 4: Figure S4F-G). Therefore, these results indicate that the observed changes in $M e c p 2 / \mathrm{MeCP} 2$ expression in response to decitabine exposure and withdrawal are not due to a shift in the number of cells deriving from male/female embryos.

Taken together, our data so far indicate that a single administration of decitabine for $48 \mathrm{~h}$ induces Mecp $2 \mathrm{e} 1 /$ MeCP2E1, MeCP2 (total) expression, whereas its withdrawal downregulates Mecp2 (total)/MeCP2 (total), Mecp $2 e 1 / \mathrm{MeCP} 2 \mathrm{E} 1$, and Mecp $2 e 2$ expression with minimal change in NSC differentiation. 
Decitabine mediates altered DNA methylation patterns at the Mecp2 regulatory elements

As mentioned earlier, DNA methylation changes at the overall $M E C P 2$ promoter and individual CpG sites within the $M E C P 2 / M e c p 2$ promoter are associated with altered $M E C P 2 / M e c p 2$ expression [2,19,20]. Therefore, we investigated whether altered expression of Mecp2 isoforms in our NSC system are associated with change in DNA methylation at the Mecp2 REs found within the Mecp 2 promoter and intron 1 . Bisulfite pyrosequencing analysis showed that decitabine treatment (D2) caused no significant change in the percentage DNA methylation at the Mecp 2 promoter R1 and R3 (Figure 6A, a, c). However, decitabine caused demethylation of all individual

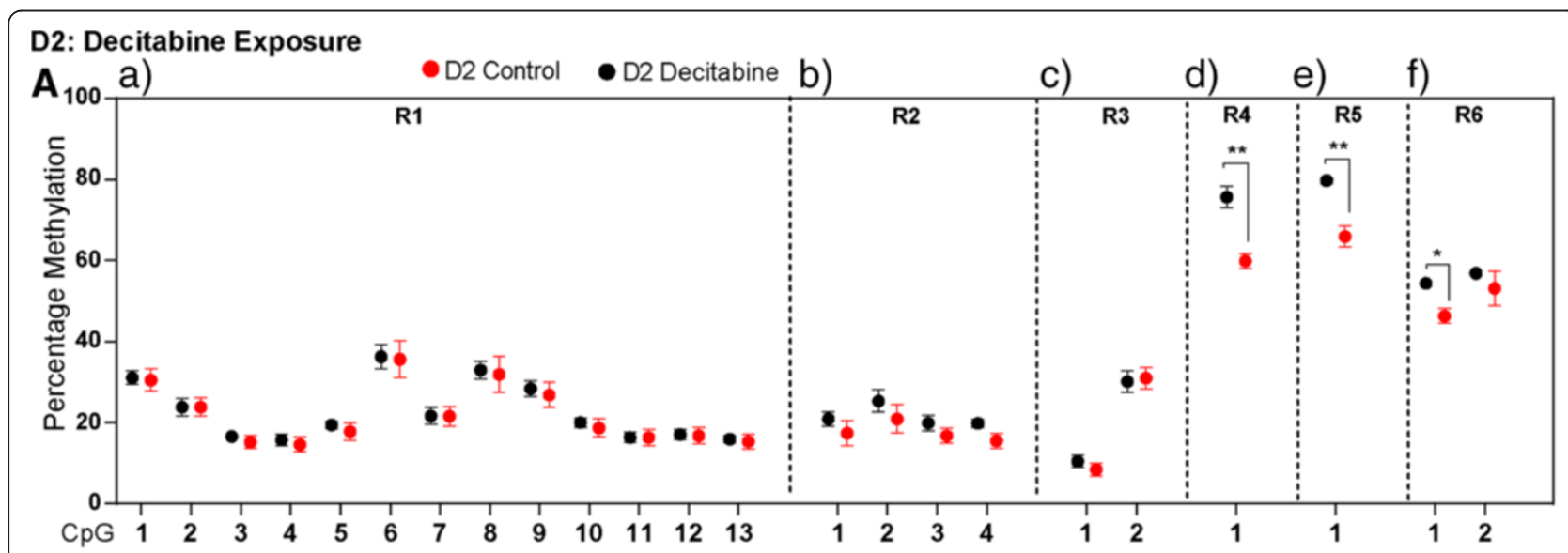

B

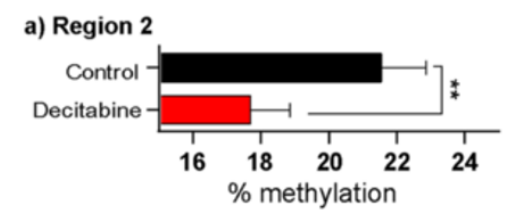

b) Intron 1

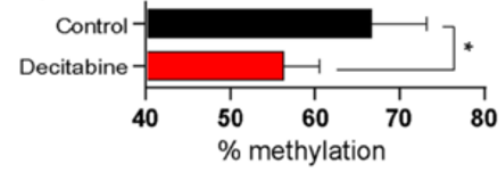

\section{D8: Decitabine Withdrawal}

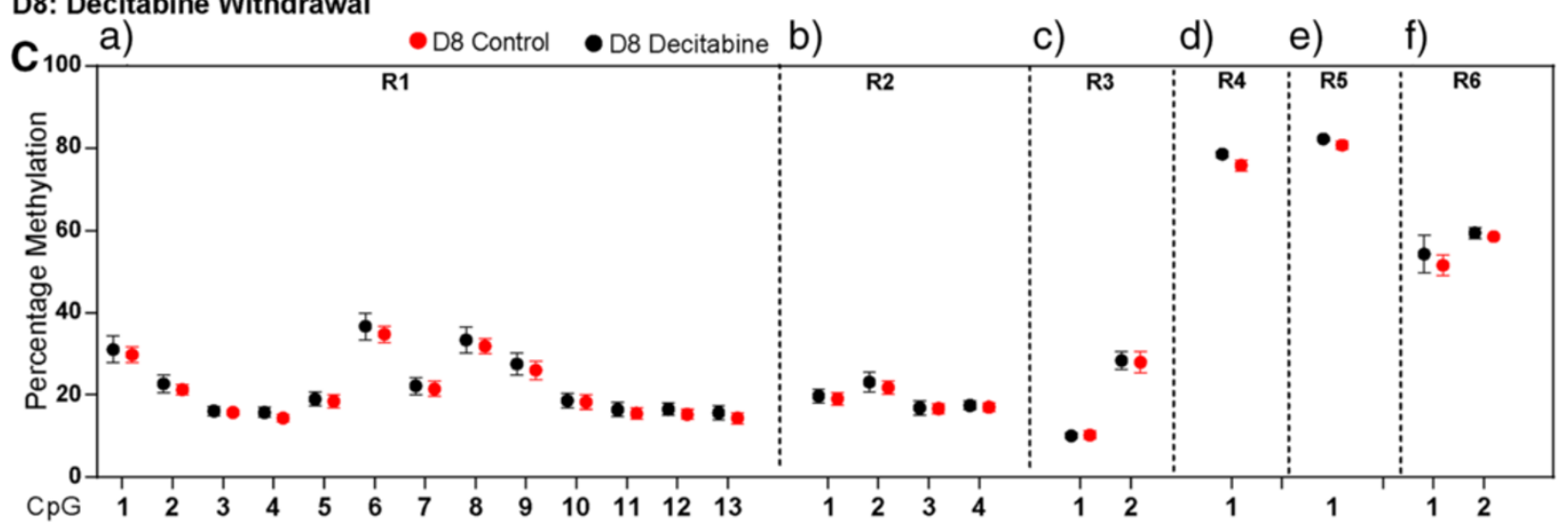

D a) Region 2

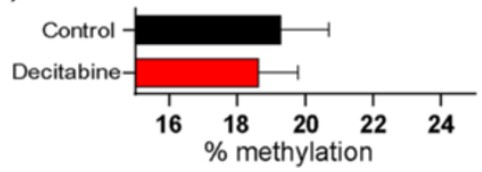

b) Intron 1

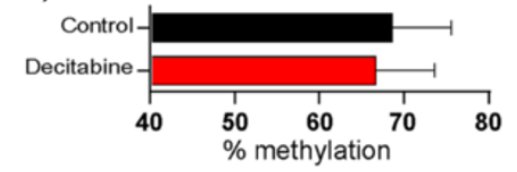

Figure 6 Bisulfite pyrosequencing analysis of DNA methylation at the Methyl CpG binding protein 2 gene (Mecp2) regulatory elements after decitabine treatment. (A) Effect of decitabine exposure at D2 on the percentage DNA methylation of Mecp2 regulatory regions. The three promoter regions are (a) R1, (b) R2, (c) R3, and the three intron 1 regions are (d) R4, (e) R5, and (f) R6. (B) Effect of decitabine exposure on average methylation over the entire region 2 (R2) (a), and intron 1 (R4 to R6) (b). Significant differences from controls: ${ }^{* * *} P<0.01 ;{ }^{*} P<0.05 ; n=3 \pm$ standard error of the mean. (C) Effect of decitabine withdrawal at D8 on percentage methylation of Mecp2 regulatory regions. The regions are promoter regions (a) R1, (b) R2 and (c) R3, and intron 1 regions (d) R4, (e) R5 and (f) R6. (D) Effect of decitabine withdrawal on average DNA methylation over entire region 2 (R2) (a), and intron 1 (R4 to R6) (b). 
CpG dinucleotides at the R2 (CpG1, 3.5\%; CpG2, 4.4\%; CpG3, 3.1\%; CpG4, 4.28\%) (Figure 6A, b), as well as the average R2 percentage DNA methylation $(3.83 \%, P<0.05)$ (Figure 6B, a). Similarly, decitabine caused demethylation of individual CpG sites at the intron $1 \mathrm{R} 4(15.8 \%, P<0.05)$, R5 (13.08\%, $P<0.05)$, and R6 (CpG1, 8.01\%, $P<0.01$; CpG2, 3.8\%, $P=0.4$ ) (Figure 6A, d-f), with significant demethylation at the entire intron $1(10.37 \%, P<0.05)$ (Figure 6B, b). These results indicated that decitabine induced significant DNA demethylation at both the Mecp2 promoter and intron 1, at the individual CpG sites and the overall DNA methylation. As mentioned earlier, this detected DNA demethylation was associated with significant upregulation of Mecp2e1 isoform, but not Mecp $2 e 2$. Therefore, it is possible that the observed changes in DNA methylation at the studied REs contribute to the upregulation of $M e c p 2 e 1$.

Similar bisulfite pyrosequencing analysis at D8 indicated that the three Mecp 2 promoter regions (R1 to R3) and intron 1 regions (R4 to R6) were remethylated and DNA methylation was almost re-established following decitabine withdrawal (Figure 6C). Analyzing the average DNA methylation over the Mecp 2 promoter R2 and the entire intron 1 (which were demethylated at D2), we observed no significant differences in DNA methylation between D8 control and decitabine-treated cells (Figure 6D). Despite the fact that DNA remethylation is expected to restore the gene expression levels, expression of both Mecp2 isoforms were significantly downregulated. This observation implies that at D8, other regulatory mechanisms apart from promoter/intron 1 DNA methylation might be involved in downregulating Mecp2 expression.

Taken together, these results show that the induced Mecp2e1 (but not Mecp2e2) expression is associated with reduced DNA methylation at the Mecp2 REs and decreased global $5 \mathrm{mC}$ DNA methylation. Hence, our findings implicate the possible role of Mecp2 genespecific DNA demethylation at the specific REs on the expression of Mecp2e1/MeCP2E1, and MeCP2 (total) at D2. Moreover, altered expression of Mecp2 isoforms without any change in DNA methylation at the Mecp2 REs at D8 imply that mechanisms other than DNA methylation could be involved in downregulating Mecp2 isoforms.

\section{Mecp2 isoform-specific expression correlates with DNA methylation at the Mecp 2 regulatory elements}

In order to establish a link between Mecp 2 isoformspecific expression and DNA methylation, we performed Pearson's correlation analysis by comparing normalized $(\log 2)$ expression of $M e c p 2$ in each dataset to the respective average percentage methylation levels over entire regions, as well as methylation at individual $\mathrm{CpG}$ sites (from both control and decitabine-treated cells).
First, we tested whether average DNA methylation of the entire Mecp 2 promoter ( $\mathrm{R} 1$ to $\mathrm{R} 3$ ), and intron 1 (R4 to R6) regions correlate with $M e c p 2 e 1$ and $M e c p 2 e 2$ expression at D2 in control and decitabine-treated cells. We observed a significant negative correlation between Mecp $2 e 1$ expression and the average methylation at R1, R3 and R5 $(r>-0.9, P<0.05)$. Correlation between Mecp2e1 upregulation and significant demethylation at R5, induced by decitabine at D2, suggests a possible contribution of R5 in upregulating Mecp2e1. On the other hand, Mecp $2 e 2$ showed significant negative correlation only with R3 methylation $(r>-0.9, P<0.05)$ (Figure 7A, a), that remained unchanged at $\mathrm{D} 2$, and this could explain the unaffected Mecp $2 e 2$ expression at D2.

Similar correlation analysis at D8 (in control D8 and decitabine-treated cells), indicated that Mecp $2 e 1$ shows a significant negative correlation with average DNA methylation at the promoter R1 $(r>-0.7, P<0.05), \mathrm{R} 2$ $(r>-0.8, P<0.05)$ and R3 $(r>-0.7, P=0.06$, close to significant) and a significant positive correlation with the average DNA methylation at the intron $1 \mathrm{R} 6 \quad(r>0.9$, $P<0.001$ ) (Figure 7A, b). In contrast, Mecp $2 e 2$ did not show any significant correlation with any of the promoter regions ( $\mathrm{R} 1$ to $\mathrm{R} 3$ ), but showed a significant positive correlation with the average methylation at intron $1 \mathrm{R} 4$ $(r>0.9, P<0.05)$ and R6 $(r>0.9, P<0.01)$. This divergence in the correlation patterns (negative and positive depending on the stage of differentiation), might imply a potential dynamic role of DNA methylation in regulating Mecp2 isoforms at different stages of NSC differentiation.

Last, we investigated whether individual CpG sites within the studied regions (R1 to R6) showed specific correlation with either Mecp2 isoform (Figure 7B-C). Implicating the possible role of promoter R1 and R2 in mainly regulating $M e c p 2 e 1$ (major isoform) at both D2 and D8, we observed a negative correlation between CpG methylation and Mecp $2 e 1$ expression at several CpG sites $(r>-0.8, P<0.05)$ (Figure $7 \mathrm{~B}$, a, and $7 \mathrm{C}$, a). At D2, unlike other REs, the average methylation over R3 showed an equally strong negative correlation with both Mecp2e1 $(r=-0.94, P<0.05)$ and Mecp2e2 $(r=-0.98$, $P<0.01)$ (Figure 7A, a). Therefore, we studied the two individual CpG sites located within R3 far apart from each other, which were differentially methylated (CpG1, approximately $10 \%$ and CpG2, approximately $30 \%$ ) (Figure 6A, c). Interestingly, CpG1 showed a significant negative correlation with Mecp2e1 $(r=-0.9, P<0.05)$, while CpG2 showed a significant negative correlation with Mecp 2e2 ( $\mathrm{r}=-0.9, P<0.01)$ (Figure $7 \mathrm{~B}, \mathrm{c})$. Further confirming the potential role of these two $\mathrm{CpG}$ sites within R3 in Mecp2 isoform-specific expression, a similar correlation (CpG1: Mecp2e1, $r=-0.85, P<0.05$; CpG2: Mecp2e2, $r=-0.87, P<0.05$ ) was observed at D8 (Figure $7 \mathrm{C}, \mathrm{c}$ ). The studied intron 1 regions seemed to have preferential 


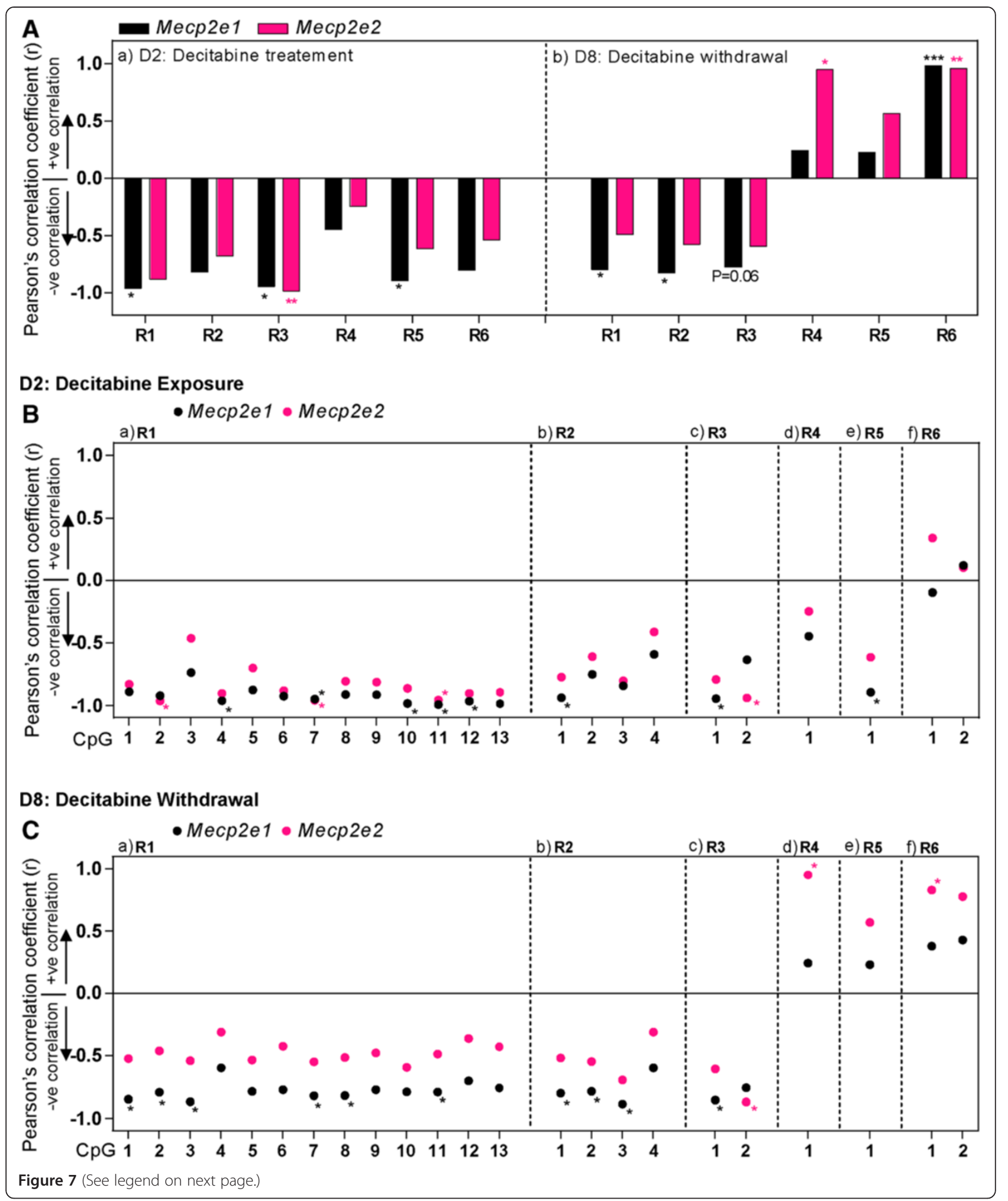


(See figure on previous page.)

Figure 7 Correlation analysis between DNA methylation at the Methyl CpG binding protein 2 gene (Mecp2) regulatory elements and Mecp2 expression after decitabine treatment (day 2) and decitabine withdrawal (day 8). All graphs represent the Pearson's correlation coefficient ( $r$ ) for Mecp2e1 (black), and Mecp2e2 (pink): statistical significance: ${ }^{* * *} P<0.001 ;{ }^{* *} P<0.01 ;{ }^{*} P<0.05 ; n=3$. (A) Correlation coefficients for the relation between Mecp2 expression and average methylation over entire regions in Mecp2 promoter (region (R)1 to R3) and intron 1 (R4 to R6) after decitabine exposure on day 2 (D2) (a), and after decitabine withdrawal on D8 (b). (B) After decitabine exposure: correlation coefficients for Mecp2el (black), and Mecp2e2 (pink) with individual CpG methylation at the promoter regions (a) R1, (b) R2 and (c) R3, and intron 1 regions (d) R4, (e) R5 and (f) R6. (C) After decitabine withdrawal: correlation coefficients for Mecp2el (black), and Mecp2e2 (pink) with individual CpG methylation at promoter regions (a) R1, (b) R2 and (c) R3, and intron 1 regions (d) R4, (e) R5 and (f) R6. Statistical significance: ${ }^{*} P<0.05$; $n=3$.

correlation with individual isoforms. For instance, at D2, the only CpG site within R5 showed negative, significant correlation with Mecp2e1 ( $r>-0.8, P<0.05)$ (Figure 7B, e). Interestingly, at D8 intron $1 \mathrm{R} 4$ and R6 showed positive, significant correlation with the Mecp $2 e 2$ isoform $(r>0.8$, $P<0.05$ ) (Figure $7 \mathrm{C}, \mathrm{d}-\mathrm{f}$ ). The observed correlations for all REs are represented in Figure $7 \mathrm{~B}-\mathrm{C}$ and are summarized in Figure 8 .

Taken together, these results show a strong $(r>0.8$, $P<0.05)$ and dynamic (positive or negative) relationship between DNA methylation at the Mecp2 REs and expression of Mecp2 isoforms depending on the different stages of NSC differentiation. Therefore, these results implicate a possible dynamic role of DNA methylation at the Mecp2 REs in regulating Mecp2 isoform-specific expression.

\section{Discussion}

In the brain, precisely controlled $M E C P 2 / \mathrm{MeCP} 2$ transcript and protein expression levels are critical, as even slightly altered expression is associated with severe neurological symptoms [2,16,57-60]. However, so far little is known about how MeCP2 expression is regulated in the developing brain. MeCP2 is a major epigenetic regulator in brain, and its reduced expression in the autistic brain is associated with $M E C P 2$ promoter hypermethylation [2]. Surprisingly, the role of DNA methylation in $\mathrm{MeCP} 2$ expression during brain development is unclear. Currently, most diseases that are associated with aberrant MeCP2 function or expression deficits, including autism and Rett syndrome, have no cure or effective treatment. This underscores an urgent need for investigating how MeCP2 expression is regulated in the brain. Such knowledge for addressing this gap is essential for designing possible future therapeutic strategies. DNA methylation is a reversible epigenetic modification [22], which can be targeted by existing Food and Drug Administration (FDA)-approved drugs, including decitabine, which is suggested for use in autism [61,62]. Therefore, investigating the effect of such epigenetic drugs on MeCP2 expression is important. Therapeutic approaches such as gene therapy or restoring MeCP2 expression by genetic engineering have been suggested as possible therapeutic strategies for $\mathrm{MeCP} 2$-associated disorders $[14,15,35]$. However, even mild MeCP2 overexpression can lead to severe neurological complications, highlighting the importance of understanding $\mathrm{MeCP} 2$ regulatory mechanisms. Since both $\mathrm{MeCP} 2$ isoforms have been implicated in severe neurological disorders, investigating MeCP2 regulation is equally important for individual isoforms. This present study is the first report on the potential role of DNA methylation at the Mecp2 REs and the impact on the expression of Mecp2 isoforms.

We observed globally altered DNA methylation upon decitabine exposure and withdrawal. Since DNA methylation is a major epigenetic mechanism that is involved in modulating gene expression and chromatin architecture [22], these observed changes in $5 \mathrm{mC}$ levels may possibly lead to altered chromatin structure and genome-wide changes in gene expression. Furthermore, the presented findings highlight that exposure to drugs that disturb the epigenetic marks during differentiation of brain cells may lead to aberrant DNA methylation profiles. Our observations at D8 indicate that, even after the disturbance factor is withdrawn from the system, an epigenetic memory for this disturbance may be associated throughout cellular differentiation of brain cells. Thus, our findings highlight the biological importance of maintaining proper regulation of epigenetic factors/modifications during brain development with a clear focus on DNA methylation and MeCP2.

Our results show that decitabine alters Mecp $2 / \mathrm{MeCP} 2$ expression at both the transcript and protein levels. Importantly, even minor changes in Mecp 2 transcript expression led to nearly 2- to 3-fold altered protein expression, highlighting the biological significance of proper regulation of $M e c p 2$ expression at the transcript levels. The observed correlation between the Mecp2/ $\mathrm{MeCP} 2$ (total) and Mecp2e1/MeCP2E1 transcript/protein expression at D2 reinforces the concept that potential changes in Mecp2 transcript levels may reflect possible changes at the protein levels. However, the noncorrelated Mecp2/MeCP2 (total) and Mecp2e1/MeCP2E1 transcript/protein expression at D8 indicates that decitabine withdrawal causes not only transcriptional but also, post-transcriptional regulation of MeCP2 expression, leading to reduced expression of MeCP2 (total)/MeCP2E1. One such post-transcriptional regulatory mechanism 


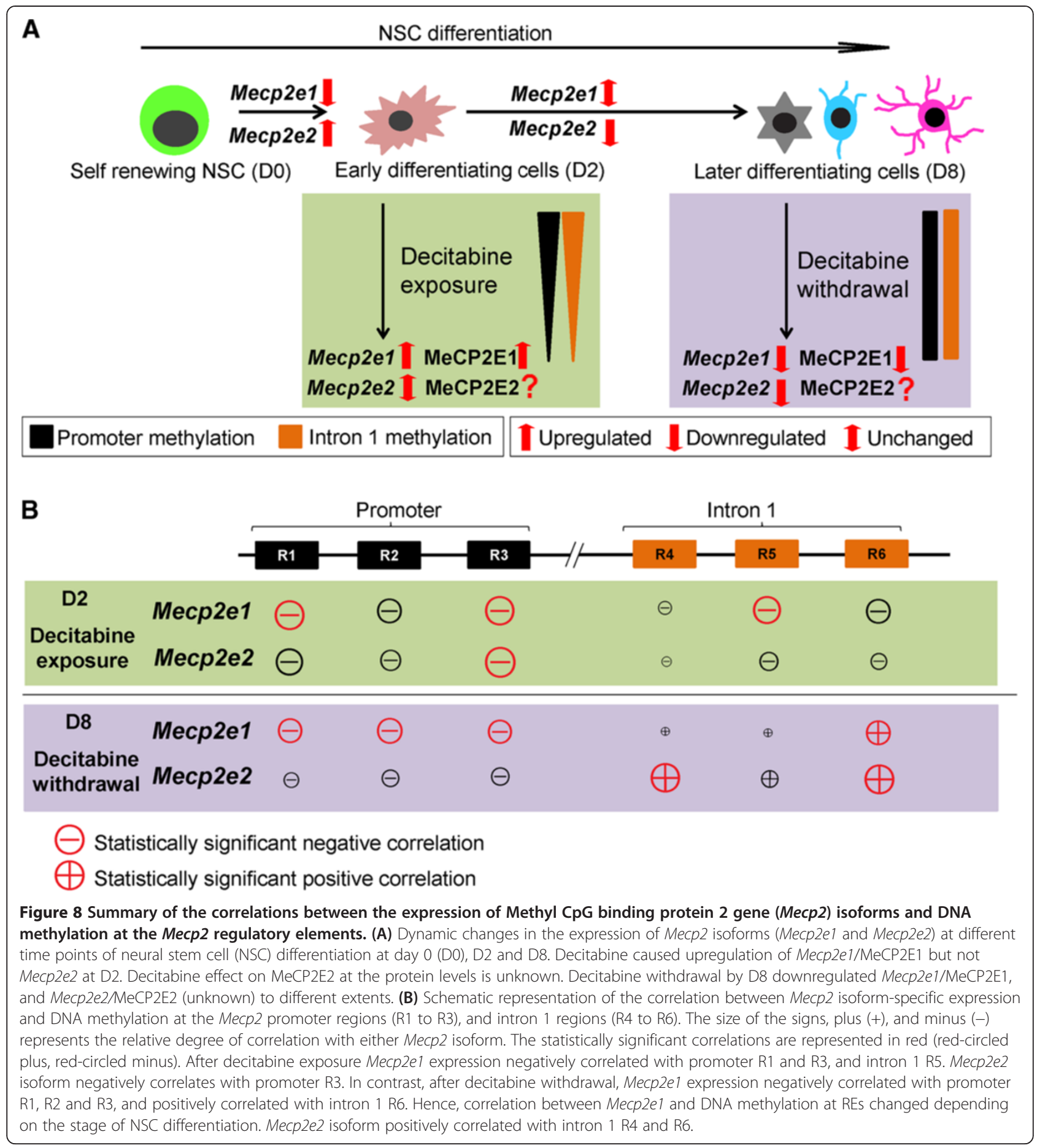

could be the action of micro-RNAs such as miR132, expression of which has been shown to be increased by 5 aza-2'-deoxycytidine/decitabine [63], and has the ability to repress MeCP2 expression [64].

Increased promoter methylation of autistic candidate genes such as RORA, BCL2 and MECP2 are shown to be associated with reduced expression of these genes in autistic patients $[2,19,62]$. Treatment with decitabine was shown to demethylate promoters and restore/induce the expression of the silenced RORA and BCL2 in autistic and patients with fragile $\mathrm{X}$ syndrome and hence, the use of DNA demethylating agents in drug therapy for autism and fragile $\mathrm{X}$ syndrome has been suggested [61,62]. A similar strategy to restore/induce MeCP2 expression might be extended to treat such diseases associated with reduced $\mathrm{MeCP} 2$ expression, including autism and RTT. 
Providing insights on such therapeutic strategies, the application of epigenetic drug therapy to induce nonmutated copy of $M E C P 2$ expression in Rett syndrome cell lines has been suggested and attempted previously [65]. Therefore, our findings on the ability of decitabine to induce $\mathrm{MeCP} 2$ expression in differentiating NSC provide further insights on designing possible drug therapies for autism. Even though the exposure of RTT cell lines (fibroblasts) to lower doses of decitabine for a longer period did not activate $M E C P 2$ expression [65], our results indicate that moderate dose of decitabine can induce Mecp2/MeCP2 expression within a shorter period. However, inhibition of MeCP2 by withdrawal of decitabine as well as other observed changes in DNA methyl marks implies that such drug therapy should be administrated with great caution.

Our findings on the changes in DNA methylation at the Mecp2 REs are in agreement with the previous reports on $M E C P 2$ promoter methylation, which demonstrate that an approximate difference of 2.0 to $2.5 \%$ overall methylation over a region -233 to -531 upstream of the $M E C P 2$ promoter is correlated with reduced MECP2 expression in autistic male brains. The authors report that within the $15 \mathrm{CpG}$ sites found in this $M E C P 2$ promoter region, two CpG sites are specifically altered in the autistic males [2]. Furthermore, our results are in agreement with a previous report on significantly reduced $\mathrm{MeCP} 2$ expression in the postnatal mouse brain (under stress), which is associated with 2 to $5 \%$ increased methylation at the individual $\mathrm{CpG}$ sites within a 164-bp region of the Mecp 2 promoter [20]. Supporting these observations, studies have also shown minor differences, such as 2 to $5 \%$ DNA methylation causing significant changes in the expression of other genes, such as RASSF1, in the human brain [66], AMOTL2 in the human heart [67], and PGC1 $\alpha$ in human muscles [68]. Therefore, although the detected DNA methylation changes in this current study are not considerably high (they varied between 2 to $15 \%$ ), they were statistically significant for average DNA methylation (within R1, R3 and R5) during NSC differentiation, and for several specific CpG dinucleotides subsequent to decitabine treatment (within R2, R4, R5, and R6), and are likely to be biologically important.

The Mecp 2 promoter CpG island studied by Franklin et al., [20] overlaps with the R1 and R2 of the Mecp2 promoter that we studied here. The significantly methylated CpGs reported in their study coincides with the R2 CpGs, where we observed changes at individual $\mathrm{CpG}$ sites as well as average methylation upon decitabine treatment. However, in our study we did not see any significant change in the R1 CpG sites (both D2 and D8), where Franklin et al., reported DNA methylation changes. Importantly, the results we obtained for one of the promoter regions studied (R2) are in agreement with this previous report, which showed a biological and functional importance of the methylation changes in regulating $\mathrm{MeCP} 2$ expression in response to stress in vivo. Therefore, it is likely that the detected changes we observed in the Mecp2 REs in our study also have biological importance. The hypermethylation of this R2 region in mouse brain was associated with MeCP2 downregulation [20], and hence it is possible that the hypomethylation/demethylation of the same $\mathrm{R} 2$ region causes Mecp2/MeCP2 upregulation.

Our results on the ability of $2.5 \mu \mathrm{M}$ decitabine to upregulate $M e c p 2 e 1$ (but not $M e c p 2 e 2$ ) suggest that the two isoforms may have different sensitivities to drugs/ chemicals. This observation is in agreement with the previous report on the higher sensitivity of Mecp $2 e 1$ than Mecp2e2 to Bisphenol A [69]. These observations further suggest that the differential sensitivity to drugs might be used to specifically induce only one Mecp 2 isoform. This is also important because overexpression of Mecp2e2, but not Mecp2e1 causes neuronal cell death [10]. Hence, our study provides a functional relevance of DNA demethylation at the Mecp2 REs by decitabine causing upregulation of Mecp2e1, but not Mecp2e2.

The observed negative correlation between the expression of both Mecp2 isoforms and Mecp2 promoter elements are novel and are in accordance with previous correlation studies on the human MECP2 expression and promoter DNA methylation [2,19]. Furthermore, our study is novel in demonstrating a dynamic (positive/ negative) correlation between the intronic DNA methylation and expression of Mecp2 isoforms in differentiating brain cells. It is possible that the promoter regions analyzed in our study (which also overlap with the core Mecp 2 promoter [13]) might be shared by both Mecp2 isoforms, whereas depending on the stage of neural differentiation, intron 1 regions may add another layer of regulation for Mecp2 isoform-specific expression. Supporting our findings, the role of intronic DNA methylation in regulating gene expression of other genes has been previously reported [70,71]. Several other reports also show evidence that gene expression negatively correlates with promoter methylation and positively correlates with gene-body methylation $[67,72]$.

Intronic DNA methylation is reported to be involved in regulating alternative splicing $[27,28]$. Although, it is known that Mecp2 isoforms are generated by alternative splicing $[4,5]$, the underlying molecular mechanisms are still unclear. We observed that the expression ratio of Mecp $2 e 1 / M e c p 2 e 2$ changed during NSC differentiation. The observed correlation between the splice ratio and intron 1 R4 DNA methylation in differentiating NSC at D2 would provide insights on the potential importance of this region in Mecp 2 alternative splicing. 
The intron 1 regions analyzed in this study were designated as part of a silencer element, which has been previously proposed to regulate $M E C P 2$ alternative splicing and tissue-specific expression [12]. Our findings are in agreement with possible involvement of these regions in Mecp2 isoform-specific expression. Although the link between DNA methylation and Mecp2 expression is supported by our results in the NSC system, the contribution of other epigenetic modifications such as histone acetylation and histone methylation should not be excluded $[73,74]$.

\section{Conclusion}

The summary of the findings presented in our study is illustrated in Figure 8. First, expression of Mecp2 isoforms was significantly and reciprocally changed at different stages of NSC differentiation, in association with minor but significant changes in DNA methylation at selected Mecp2 REs, suggesting possible involvement of these regions in Mecp 2 regulation. Second, treatment of differentiating NSC with decitabine for $48 \mathrm{~h}$ led to demethylation of specific Mecp2 REs (promoter R2 and all intron 1 regions) and subsequent upregulation of Mecp2e1/MeCP2E1 (but not Mecp2e2), implying the differential sensitivity of the two Mecp 2 isoforms to decitabine. Such differential sensitivity of Mecp2 isoforms to decitabine might be useful in future drug therapies to specifically activate one isoform but not the other. Furthermore, the ability of decitabine to induce Mecp2e1/ MeCP2E1 at both transcript and protein levels provide insights for future therapeutic strategies for MeCP2 deficiency-related neurodevelopmental disorders such as autism and Rett syndrome. Finally, the significant and dynamic (positive or negative) correlation between the expression of Mecp2 isoforms and DNA methylation implies the potential contribution of these REs in regulating Mecp2 isoforms at different stages of neural differentiation. Collectively, our study contributes to the understanding of expression and regulation of Mecp 2 isoforms during neural development and provides important insights for future therapeutic applications of decitabine for MeCP2-related neurological disorders.

\section{Additional files}

Additional file 1: Figure S1. Comparison of $\mathrm{CpG}$ sites in human Methy CpG binding protein 2 gene (MECP2) and mouse Methyl CpG binding protein 2 gene (Mecp2) promoter and intron 1. CpG sites analyzed in the mouse Mecp2 (black) are underlined. Conserved CpGs between mouse and human sequences are also underlined in human MECP2 (red) sequence.

Additional file 2: Figure S2. Detection of KI67 in day 2 (D2) control cells. Kl67 was detected in $(98.8 \% \pm 0.8)$ of the D2 cell population, indicating that they were actively proliferating. Scale bars represent $20 \mu \mathrm{m}$.
Additional file 3: Figure S3. Relationship between the ratio of mouse Methyl CpG binding protein 2 gene Mecp2 splice variants and DNA methylation at selected Mecp2 regulatory elements. Pearson's correlation analysis between DNA methylation at the Mecp2 regions R1, R4 and R5 and Mecp2e1/Mecp2e2 ratio at different stages of neural stem cell (NSC) differentiation. Significant differences: ${ }^{*} P<0.05$. The regions are, promoter regions R1: CpG island contains $13 \mathrm{CpG}$ sites, intron 1 regions $\mathrm{R} 4: 1 \mathrm{CpG}$ site, and R5: 1 CpG site; $n=3 \pm$ standard error of the mean.

Additional file 4: Figure S4. Determination of the male/female contribution at different stages of neural stem cell (NSC) differentiation. (A) PCR amplification of Sry (402 bp) and //3 (544 bp) in adult male cortex (positive control) and the absence of the signal in negative control PCR (no template). (B) The detection of Sry and //3 in the positive and negative controls and during NSC differentiation (day 0 (D0), D2, D8). The graph represents ratio of Sry/II3; $n=3 \pm$ standard error of the mean (SEM). (C) Expression of Xist transcripts relative to Gapdh at different stages of NSC differentiation; $n=3 \pm$ SEM. Significance was determined at ${ }^{*} P<0.05$. (D) Ratio of Sry/l13 in D2 control and D2 decitabine-treated cells. (E) Ratio of Sry/II3 in control and decitabine-treated cells at D8; $n=3 \pm$ SEM. Expression of Xist transcripts relative to Gapdh at D2 after decitabine treatment $(F)$, and at D8 after decitabine withdrawal $(G) ; n=3 \pm$ SEM. Significance was determined at $* P<0.05$.

\section{Abbreviations}

5hmC: 5-hydroxymethylcytosine; 5mC: 5-methylcytosine; ANOVA: Analysis of variance; bFGF: Basic Fibroblast growth factor; bp: Base pairs; CNPase: 2',3'Cyclic-nucleotide 3'-phosphodiesterase; D: Day; DAPI: 4',6-diamidino-2phenylindole; DMEM: Dulbecco's modified Eagle's medium; DNMT: DNA methyltransferases; E: Embryonic day; GAPDH: Glyceraldehyde-3-phosphate dehydrogenase; GFAP: Glial fibrillary acidic protein; IF: Immunofluorescence; MBP: Myelin basic protein; MECP2: Methyl CpG binding protein 2, human gene; Mecp2: Methyl CpG binding protein 2, mouse gene; MeCP2: Methyl CpG binding protein; NEUN: NEUronal Nuclei; NSC: Neural stem cells; OLIG2: Oligodendrocyte lineage transcription factor 2; qRT-PCR: Quantitative reverse transcription polymerase chain reaction; $r$ : Pearson's correlation coefficient; RE: Regulatory element; RTT: Rett syndrome; rhEGF: Recombinant human epidermal growth factor; SEM: Standard error of the mean; TUB III: TUBULIN III; WB: Western blot.

\section{Competing interests}

The authors have declared that no competing interests exist.

\section{Author contributions}

VRBL, RMZ and MR conceived and designed experiments. MR performed neural stem cell isolation, culture and differentiation. RMZ maintained neural stem cell cultures. VRBL performed qRT-PCR, dot blot, WB, IF, inverted microscopy imaging. RMZ performed IF and confocal imaging. VRBL and MR analyzed data. MR contributed reagents/materials/analysis tools. VRBL and MR wrote the paper. All authors read and approved the final manuscript.

\section{Acknowledgements}

We thank Mr Carl Olson in the Rastegar laboratory for neurosphere sectioning. This work was supported by funds from the Natural Sciences and Engineering Research Council of Canada (NSERC Discovery Grant 3724052009), and Scottish Rite Charitable Foundation of Canada (SRCFC, Grant 10110). VRBL and RMZ are recipients of MHRC-MICH studentship awards. The NESTIN monoclonal antibody developed by Susan Hockfield was obtained from the Developmental Studies Hybridoma Bank, developed under the auspices of the NICHD and maintained by The University of lowa, Department of Biology, lowa City, IA 52242.

Received: 11 June 2013 Accepted: 1 October 2013 Published: 15 November 2013

\section{References}

1. Zachariah RM, Rastegar M: Linking epigenetics to human disease and Rett syndrome: the emerging novel and challenging concepts in MeCP2 research. Neural plast 2012, 2012:415825. 
2. Nagarajan RP, Hogart AR, Gwye Y, Martin MR, LaSalle JM: Reduced MeCP2 expression is frequent in autism frontal cortex and correlates with aberrant MECP2 promoter methylation. Epigenetics 2006, 1:e1-e11.

3. Amir RE, Van den Veyver IB, Wan M, Tran CQ, Francke U, Zoghbi HY: Rett syndrome is caused by mutations in X-linked MECP2, encoding methylCpG-binding protein 2. Nat Genet 1999, 23:185-188.

4. Kriaucionis S, Bird A: The major form of MeCP2 has a novel N-terminus generated by alternative splicing. Nucleic Acids Res 2004, 32:1818-1823.

5. Mnatzakanian GN, Lohi H, Munteanu I, Alfred SE, Yamada T, MacLeod PJ, Jones JR, Scherer SW, Schanen NC, Friez MJ, Vincent JB, Minassian BA: A previously unidentified MECP2 open reading frame defines a new protein isoform relevant to Rett syndrome. Nat Genet 2004, 36:339-341.

6. Dragich JM, Kim YH, Arnold AP, Schanen NC: Differential distribution of the MeCP2 splices variants in the postnatal mouse brain. J Comp Neurol 2007, 501:526-542.

7. Zachariah RM, Olson CO, Ezeonwuka C, Rastegar M: Novel MeCP2 isoformspecific antibody reveals the endogenous MeCP2E1 expression in murine brain, primary neurons and astrocytes. PloS one 2012, 7:e49763.

8. Saunders CJ, Minassian BE, Chow EW, Zhao W, Vincent JB: Novel exon 1 mutations in MECP2 implicate isoform MeCP2_e1 in classical Rett syndrome. Am J Med Genet Part A 2009, 149A:1019-1023.

9. Fichou Y, Nectoux J, Bahi-Buisson N, Rosas-Vargas H, Girard B, Chelly J, Bienvenu T: The first missense mutation causing Rett syndrome specifically affecting the MeCP2_e1 isoform. Neurogenet 2009, 10:127-133.

10. Dastidar SG, Bardai FH, Ma C, Price V, Rawat V, Verma P, Narayanan V, D'Mello SR: Isoform-specific toxicity of Mecp2 in postmitotic neurons: suppression of neurotoxicity by FoxG1. J Neurosci 2012, 32:2846-2855.

11. Williamson SL, Christodoulou J: Rett syndrome: new clinical and molecular insights. Eur J Hum Genet 2006, 14:896-903.

12. Liu J, Francke U: Identification of cis-regulatory elements for MECP2 expression. Hum Mol Genet 2006, 15:1769-1782.

13. Adachi M, Keefer EW, Jones FS: A segment of the Mecp2 promoter is sufficient to drive expression in neurons. Hum Mol Genet 2005, 14:3709-3722

14. Jugloff DG, Vandamme K, Logan R, Visanji NP, Brotchie JM, Eubanks JH: Targeted delivery of an Mecp2 transgene to forebrain neurons improves the behavior of female Mecp2-deficient mice. Hum Mol Genet 2008, 17:1386-1396.

15. Kerr B, Soto CJ, Saez M, Abrams A, Walz K, Young Jl: Transgenic complementation of MeCP2 deficiency: phenotypic rescue of Mecp2-null mice by isoform-specific transgenes. Eur J Hum Genet 2012, 20:69-76.

16. Collins AL, Levenson JM, Vilaythong AP, Richman R, Armstrong DL, Noebels $J$, David Sweatt J, Zoghbi HY: Mild overexpression of MeCP2 causes a progressive neurological disorder in mice. Hum Mol Genet 2004, 13:2679-2689.

17. Luikenhuis S, Giacometti E, Beard CF, Jaenisch R: Expression of MeCP2 in postmitotic neurons rescues Rett syndrome in mice. Proc Nat Acad Sci USA 2004, 101:6033-6038.

18. Singh J, Saxena A, Christodoulou J, Ravine D: MECP2 genomic structure and function: insights from ENCODE. Nucleic Acids Res 2008, 36:6035-6047.

19. Nagarajan RP, Patzel KA, Martin M, Yasui DH, Swanberg SE, Hertz-Picciotto I, Hansen RL, Van de Water J, Pessah IN, Jiang R, Robinson WP, LaSalle JM: MECP2 promoter methylation and $\mathrm{X}$ chromosome inactivation in autism. Autism Res 2008, 1:169-178.

20. Franklin TB, Russig H, Weiss IC, Graff J, Linder N, Michalon A, Vizi S, Mansuy IM: Epigenetic transmission of the impact of early stress across generations. Biol Psych 2010, 68:408-415.

21. Delcuve GP, Rastegar M, Davie JR: Epigenetic control. J Cell Phys 2009, 219:243-250.

22. Liyanage VRB, Zachariah RM, Delcuve GP, Davie JR, Rastegar M: New Developments in Chromatin Research: An Epigenetic Perspective. In New Developments in Chromatin Research. Edited by Simpson NM, Stewart VJ. NY, USA: Nova Science Publishers; 2012:29-58.

23. Jeltsch A: Beyond Watson and Crick: DNA methylation and molecular enzymology of DNA methyltransferases. Chembiochem 2002, 3:274-293.

24. Kriaucionis S, Heintz N: The nuclear DNA base 5-hydroxymethylcytosine is present in Purkinje neurons and the brain. Science 2009, 324:929-930.

25. Tahiliani M, Koh KP, Shen Y, Pastor WA, Bandukwala H, Brudno Y, Agarwal S, lyer LM, Liu DR, Aravind L, Rao A: Conversion of 5-methylcytosine to 5hydroxymethylcytosine in mammalian DNA by MLL partner TET1. Science 2009, 324:930-935.
26. Elango N, Yi SV: DNA methylation and structural and functional bimodality of vertebrate promoters. Mol Biol Evol 2008, 25:1602-1608.

27. Flores K, Wolschin F, Corneveaux JJ, Allen AN, Huentelman MJ, Amdam GV: Genome-wide association between DNA methylation and alternative splicing in an invertebrate. BMC genomics 2012, 13:480.

28. Shukla S, Kavak E, Gregory M, Imashimizu M, Shutinoski B, Kashlev M Oberdoerffer P, Sandberg R, Oberdoerffer S: CTCF-promoted RNA polymerase II pausing links DNA methylation to splicing. Nature 2011, 479:74-79.

29. Mossman D, Kim KT, Scott RJ: Demethylation by 5-aza-2'-deoxycytidine in colorectal cancer cells targets genomic DNA whilst promoter CpG island methylation persists. BMC cancer 2010, 10:366.

30. Ishimaru N, Fukuchi M, Hirai A, Chiba Y, Tamura T, Takahashi N, Tabuchi A, Tsuda M, Shiraishi M: Differential epigenetic regulation of BDNF and NT-3 genes by trichostatin A and 5-aza-2'-deoxycytidine in Neuro-2a cells. Biochem Biophys Res Comm 2010, 394:173-177

31. Bazan E, Alonso FJ, Redondo C, Lopez-Toledano MA, Alfaro JM, Reimers D, Herranz AS, Paino CL, Serrano AB, Cobacho N, Caso E, Lobo MV: In vitro and in vivo characterization of neural stem cells. Histol Histopathol 2004, 19:1261-1275

32. Tropepe V, Sibilia M, Ciruna BG, Rossant J, Wagner EF, van der Kooy D: Distinct neural stem cells proliferate in response to EGF and FGF in the developing mouse telencephalon. Dev Biol 1999, 208:166-188.

33. Bartl J, Mori T, Riederer P, Ozawa H, Grunblatt E: Methylphenidate enhances neural stem cell differentiation. J Mol Psych 2013, 1:5.

34. Kishi N, Macklis JD: MECP2 is progressively expressed in post-migratory neurons and is involved in neuronal maturation rather than cell fate decisions. Mol Cell Neurosci 2004, 27:306-321.

35. Rastegar M, Hotta A, Pasceri P, Makarem M, Cheung AY, Elliott S, Park KJ, Adachi M, Jones FS, Clarke ID, Dirks P, Ellis J: MECP2 isoform-specific vectors with regulated expression for Rett syndrome gene therapy. Plos one 2009, 4:e6810.

36. Olynik BM, Rastegar M: The genetic and epigenetic journey of embryonic stem cells into mature neural cells. Frontiers in genetics 2012, 3:81.

37. Barber BA, Liyanage VR, Zachariah RM, Olson CO, Bailey MA, et al.: Dynamic expression of MEIS1 homeoprotein in E14.5 forebrain and differentiated forebrain-derived neural stem cells. Ann Anat 2013, 195:431-440.

38. Lambert JF, Benoit BO, Colvin GA, Carlson J, Delville Y, Quesenberry PJ: Quick sex determination of mouse fetuses. J Neurosci Methods 2000, 95:127-132.

39. Hartshorn C, Rice JE, Wangh L: Developmentally-regulated changes of Xist RNA levels in single preimplantation mouse embryos, as revealed by quantitative real-time PCR. Mol Reprod Dev 2002, 61:425-436.

40. Kobrossy L, Rastegar M, Featherstone M: Interplay between chromatin and trans-acting factors regulating the Hoxd4 promoter during neural differentiation. J Biol Chem 2006, 281:25926-25939.

41. Nolte C, Rastegar M, Amores A, Bouchard M, Grote D, Maas R, Kovacs EN, Postlethwait J, Rambaldi I, Rowan S, Yan YL, Zhang F, Featherstone M: Stereospecificity and PAX6 function direct Hoxd4 neural enhancer activity along the antero-posterior axis. Dev Biol 2006, 299:582-593.

42. Huang H, Rastegar M, Bodner C, Goh SL, Rambaldi I, Featherstone M: MEIS $\mathrm{C}$ termini harbor transcriptional activation domains that respond to cell signaling. J Biol Chem 2005, 280:10119-10127.

43. Rastegar M, Kobrossy L, Kovacs EN, Rambaldi I, Featherstone M: Sequential histone modifications at Hoxd4 regulatory regions distinguish anterior from posterior embryonic compartments. $\mathrm{Mol}$ Cel Biol 2004, 24:8090-8103.

44. Manczak M, Mao P, Nakamura K, Bebbington C, Park B, Reddy PH: Neutralization of granulocyte macrophage colony-stimulating factor decreases amyloid beta 1-42 and suppresses microglial activity in a transgenic mouse model of Alzheimer's disease. Hum Mol Genet 2009, 18:3876-3893

45. Tsoporis JN, Marks A, Haddad A, Dawood F, Liu PP, Parker TG: S100B expression modulates left ventricular remodeling after myocardial infarction in mice. Circulation 2005, 111:598-606.

46. Schneider $L$, d'Adda di Fagagna F: Neural stem cells exposed to BrdU lose their global DNA methylation and undergo astrocytic differentiation. Nucleic Acids Res 2012, 40:5332-5342.

47. Mack JT, Beljanski V, Soulika AM, Townsend DM, Brown CB, Davis W, Tew KD: "Skittish" Abca2 knockout mice display tremor, hyperactivity, and abnormal myelin ultrastructure in the central nervous system. Mol Cell Biol 2007, 27:44-53. 
48. Ghoshal K, Datta J, Majumder S, Bai S, Kutay H, Motiwala T, Jacob ST: 5-Azadeoxycytidine induces selective degradation of DNA methyltransferase 1 by a proteasomal pathway that requires the KEN box, bromo-adjacent homology domain, and nuclear localization signal. Mol Cell Biol 2005, 25:4727-4741

49. Rastegar M, Rousseau GG, Lemaigre FP: CCAAT/enhancer-binding proteinalpha is a component of the growth hormone-regulated network of liver transcription factors. Endocrinol 2000, 141:1686-1692.

50. Wu CH, Rastegar M, Gordon J, Safa AR: Beta(2)-microglobulin induces apoptosis in HL-60 human leukemia cell line and its multidrug resistant variants overexpressing MRP1 but lacking Bax or overexpressing P-glycoprotein. Oncogene 2001, 20:7006-7020.

51. Gordon J, Wu CH, Rastegar M, Safa AR: Beta2-microglobulin induces caspase-dependent apoptosis in the CCRF-HSB-2 human leukemia cell line independently of the caspase- $3,-8$ and -9 pathways but through increased reactive oxygen species. Int J Cancer 2003, 103:316-327.

52. Ko M, Huang Y, Jankowska AM, Pape UJ, Tahiliani M, Bandukwala HS, An J, Lamperti ED, Koh KP, Ganetzky R, Liu XS, Aravind L, Agarwal S, Maciejewski JP, Rao A: Impaired hydroxylation of 5-methylcytosine in myeloid cancers with mutant TET2. Nature 2010, 468:839-843.

53. Choufani S, Shapiro JS, Susiarjo M, Butcher DT, Grafodatskaya D, Lou Y, Ferreira JC, Pinto D, Scherer SW, Shaffer LG, Coullin P, Caniggia I, Beyene J, Slim R, Bartolomei MS, Weksberg R: A novel approach identifies new differentially methylated regions (DMRs) associated with imprinted genes. Genome Res 2011, 21:465-476.

54. Nandakumar V, Vaid M, Katiyar SK: (-)-Epigallocatechin-3-gallate reactivates silenced tumor suppressor genes, Cip1/p21 and p16INK4a, by reducing DNA methylation and increasing histones acetylation in human skin cancer cells. Carcinogen 2011, 32:537-544.

55. Liu Q, Yang L, Gong C, Tao G, Huang H, Liu J, Zhang H, Wu D, Xia B, Hu G, Wang K, Zhuang Z: Effects of long-term low-dose formaldehyde exposure on global genomic hypomethylation in 16HBE cells. Toxicol Lett 2011, 205:235-240.

56. Christman JK: 5-Azacytidine and 5-aza-2'-deoxycytidine as inhibitors of DNA methylation: mechanistic studies and their implications for cancer therapy. Oncogene 2002, 21:5483-5495.

57. Squillaro T, Alessio N, Cipollaro M, Melone MA, Hayek G, Renieri A, Giordano A, Galderisi U: Reduced expression of MECP2 affects cell commitment and maintenance in neurons by triggering senescence: new perspective for Rett syndrome. Mol Biol Cell 2012, 23:1435-1445.

58. Smrt RD, Eaves-Egenes J, Barkho BZ, Santistevan NJ, Zhao C, Aimone JB, Gage FH, Zhao X: Mecp2 deficiency leads to delayed maturation and altered gene expression in hippocampal neurons. Neurobiol Dis 2007, 27:77-89.

59. Bodda C, Tantra M, Mollajew R, Arunachalam JP, Laccone FA, Can K, Rosenberger A, Mironov SL, Ehrenreich H, Mannan AU: Mild Overexpression of Mecp2 in Mice Causes a Higher Susceptibility toward Seizures. Am J Pathol 2013, 183:195-210.

60. Na ES, Nelson ED, Adachi M, Autry AE, Mahgoub MA, Kavalali ET, Monteggia LM: A mouse model for MeCP2 duplication syndrome: MeCP2 overexpression impairs learning and memory and synaptic transmission. J Neurosci 2012, 32:3109-3117.

61. Chiurazzi P, Pomponi MG, Willemsen R, Oostra BA, Neri G: In vitro reactivation of the FMR1 gene involved in fragile $X$ syndrome. Hum Mol Genet 1998, 7:109-113.

62. Nguyen A, Rauch TA, Pfeifer GP, Hu WW: Global methylation profiling of lymphoblastoid cell lines reveals epigenetic contributions to autism spectrum disorders and a novel autism candidate gene, RORA, whose protein product is reduced in autistic brain. FASEB J 2010, 24:3036-3051.

63. Im HI, Hollander JA, Bali P, Kenny PJ: MeCP2 controls BDNF expression and cocaine intake through homeostatic interactions with microRNA212. Nat Neurosci 2010, 13:1120-1127.

64. Feng J, Nestler EJ: MeCP2 and drug addiction. Nat Neurosci 2010, 13:1039-1041.

65. Yu D, Sakurai F, Corey DR: Clonal Rett Syndrome cell lines to test compounds for activation of wild-type MeCP2 expression. Bioorgan Med Chem Lett 2011, 21:5202-5205.

66. Mill J, Tang T, Kaminsky Z, Khare T, Yazdanpanah S, Bouchard L, Jia P Assadzadeh A, Flanagan J, Schumacher A, Wang SC, Petronis A: Epigenomic profiling reveals DNA-methylation changes associated with major psychosis. Am J Hum Genet 2008, 82:696-711.
67. Movassagh M, Choy MK, Goddard M, Bennett MR, Down TA, Foo RS: Differential DNA methylation correlates with differential expression of angiogenic factors in human heart failure. PloS one 2010, 5:e8564.

68. Barres R, Osler ME, Yan J, Rune A, Fritz T, Caidahl K, Krook A, Zierath JR Non-CpG methylation of the PGC-1alpha promoter through DNMT3B controls mitochondrial density. Cell Metab 2009, 10:189-198.

69. Warita K, Mitsuhashi T, Ohta K, Suzuki S, Hoshi N, Miki T, Takeuchi Y: Gene expression of epigenetic regulatory factors related to primary silencing mechanism is less susceptible to lower doses of bisphenol A in embryonic hypothalamic cells. J Toxico/ Sci 2013, 38:285-289.

70. Jowaed A, Schmitt I, Kaut O, Wullner U: Methylation regulates alphasynuclein expression and is decreased in Parkinson"s disease patients brains. J Neurosci 2010, 30:6355-6359.

71. Zhang X, Wu M, Xiao H, Lee MT, Levin L, Leung YK, Ho SM: Methylation of a single intronic CpG mediates expression silencing of the PMP24 gene in prostate cancer. Prostate 2010, 70:765-776.

72. Ball MP, Li JB, Gao Y, Lee JH, LeProust EM, Park IH, Xie B, Daley GQ, Church GM: Targeted and genome-scale strategies reveal gene-body methylation signatures in human cells. Nat Biotechnol 2009, 27:361-368.

73. Takebayashi S, Nakao M, Fujita N, Sado T, Tanaka M, Taguchi H, Okumura K: 5-Aza-2'-deoxycytidine induces histone hyperacetylation of mouse centromeric heterochromatin by a mechanism independent of DNA demethylation. Biochem Biophys Res Comm 2001, 288:921-926.

74. Nguyen CT, Weisenberger DJ, Velicescu M, Gonzales FA, Lin JC, Liang G, Jones PA: Histone H3-lysine 9 methylation is associated with aberrant gene silencing in cancer cells and is rapidly reversed by 5-aza-2'-deoxycytidine. Cancer Res 2002, 62:6456-6461.

doi:10.1186/2040-2392-4-46

Cite this article as: Liyanage et al: Decitabine alters the expression of Mecp2 isoforms via dynamic DNA methylation at the Mecp2 regulatory elements in neural stem cells. Molecular Autism 2013 4:46

\section{Submit your next manuscript to BioMed Central and take full advantage of:}

- Convenient online submission

- Thorough peer review

- No space constraints or color figure charges

- Immediate publication on acceptance

- Inclusion in PubMed, CAS, Scopus and Google Scholar

- Research which is freely available for redistribution 